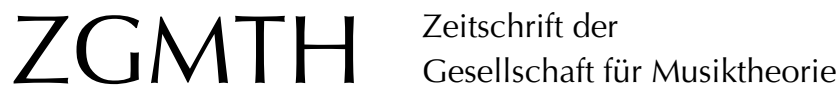

Haas, Bernhard (2011): Zu zwei Bartók-Analysen von Albert Simon. ZGMTH 8/2, 299-334. https://doi.org/10.31751/640

\section{(C) 2011 Bernhard Haas}

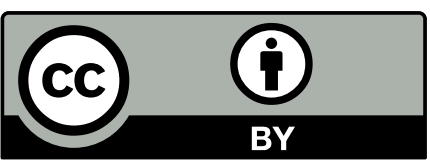

Dieser Text erscheint im Open Access und ist lizenziert unter einer Creative Commons Namensnennung 4.0 International Lizenz.

This is an open access article licensed under a

Creative Commons Attribution 4.0 International License.

veröffentlicht / first published: 17/11/2011

zuletzt geändert / last updated: 02/07/2013 


\title{
Zu zwei Bartók-Analysen von Albert Simon
}

\author{
Bernhard Haas
}

ABSTRACT: Dem ungarischen Dirigenten Albert Simon war es als Musiktheoretiker darum zu tun, zu zeigen, warum und in welcher Weise die Musik Bartóks istimmts, obwohl sie sich weder der traditionellen Tonalität noch etwa Schönbergs Zwölftontechnik bedient. Zu diesem Zweck entwickelte Simon seine Theorie der Tonfelder. Zwei Kompositionen aus dem Mikrokosmos Staccato und Geteilte Arpeggien werden analysiert, indem ihre Teile aus verschiedenen Perspektiven betrachtet werden. So lassen sich diese Kompositionen als ein Spiel von Eindeutigkeit und sehr präzis angelegter Mehrdeutigkeit lesen. Hierauf aufbauend wird versucht zu rekonstruieren, welche Tonfelder je die Einheit dieser Stücke herstellen (`Einheit‘ etwa im Sinne des Schenkerschen Ursatzes in der älteren Musik verstanden). In dieser Konstruktion offenbart sich Bartóks Definition des Verhältnisses der virtuell unendlichen Quintenreihe zur Zwölfstufigkeit sowie seine Stellung zum Verhältnis von lokalisierten zu nicht lokalisierten Tonfeldern.

Im Folgenden soll an zwei Kompositionen von Béla Bartók gezeigt werden, wie allgemeine Strukturen von Tönen bis in die feinsten Einzelheiten einer Komposition fortwirken und diese Einzelheiten bestimmen können. Die sallgemeinen Strukturen von Tönen`, um die es hier gehen soll, sind die beiden Tonfelder des Ganzen (einer Komposition). ${ }^{1}$ Diese erlauben einen unmittelbaren Zugriff aufs Werk als Ganzes; zugleich werden aus ihnen heraus viele Einzelheiten der Ausführung verständlich. ${ }^{2}$ Um diese These durchführen zu können, werden hier zuerst zwei Bartók-Analysen von Albert Simon in Auszügen vorgestellt. Die Analysen beziehen sich auf Staccato und Geteilte Arpeggien, Nr. 124 und 143 aus dem Mikrokosmos. Sie werden in der Paul Sacher Stiftung, Sammlung Paul Sacher in Basel im Magazin unter der Signatur PSS 76-142 aufbewahrt. ${ }^{3}$ Die Analysen wurden im April 1985 von Paul Sacher der Stiftung übergeben ${ }^{4}$, stellen also Simons Theorie auf dem Stand der ersten Hälfte der achtziger Jahre dar. Ich danke Herrn Dr. Felix Meyer, dem Direktor der Paul Sacher Stiftung, sehr herzlich dafür, dass sie mir diese Analysen zugänglich gemacht haben.

1 Zu diesem Begriff vgl. Haas 2004, 37-41, 46, 48, 62, 65.

2 Dieser Begriff erinnert daher ein wenig an den ıUrsatzı, den Heinrich Schenker in Hinblick auf ältere Musik ausgearbeitet hat.

3 Dort liegt außerdem eine Umschrift von mir mit zahlreichen erläuternden Fußnoten. - Unter der Signatur PSS 76-141 befindet sich ein weiteres Typoskript von Albert Simon: Die Eigentümlichkeiten der Bartókschen Musik anhand seines Werkes: Musik für Saiteninstrumente, Schlagzeug und Celesta.

4 Es ist unbekannt, wie viel Zeit zwischen der Herstellung der Analyse und ihrer Übergabe an die Paul Sacher Stiftung verstrichen ist. 
Die Texte von Albert Simon erwiesen sich als zum Teil nicht ganz leicht verständlich. Zu der Schwierigkeit, die in Simons oft eigenwilligem deutschen Sprachgebrauch liegt, kam die andere hinzu, dass oft erst durch genaues Durchdenken zu ermitteln war, auf welches Detail der Partitur gerade Bezug genommen wird. Ich habe die Auszüge zurückhaltend redigiert und versucht, die originale Diktion beizubehalten. ${ }^{5}$ Sämtliche Fußnoten zu den Auszügen aus Simons Analysen sind von mir; sie klären einerseits den Bezug auf die jeweiligen Passagen bei Bartók und erläutern andererseits Besonderheiten der Terminologie. Die Beispiele sind nicht von der Hand Albert Simons, sondern von einem bislang nicht identifizierten Schüler oder Mitarbeiter geschrieben. Es war Simons Gepflogenheit, seine oft schwer lesbaren Skizzen abschreiben zu lassen. Zunächst also Auszüge aus zwei Analysen von Albert Simon.

\section{Staccato (Mikrokosmos Nr. 124)}

»Eine Analyse beweist einerseits die Anwesenheit der entscheidenden Geschehnisse der Organisation.

Hier spielen [...] die impliziten Hinweise - die Entsprechungen/Zusammenhänge -, also die in welchem Sinne auch immer zusammengehörenden Töne, eine ebenso große Rolle wie die expliziten, offenen Aussagen. ${ }^{6}$ Die verschiedensten Arten von Geschehnissen bilden das sehr starke [...] unsichtbare Netzwerk der Schöpfung. ${ }^{7}$ Diese unauffälligen Erwähnungen, Nachdrücke und Betonungen ermöglichen es, im Ablauf des Werkes die späteren Hinweise und das letztendliche Summieren eines Ganzen zu erfassen.

Hinter einer Idee, [...] hinter einem scheinbaren Einfall, müssen - wie immer bei Bartók - Ordnungsprinzipien liegen. $\ll^{8}-$

»Wir finden zwei repetierende Töne: es und a (T. 1-10, 12-19), ein wie eine Periode beginnendes Motiv (T. 4) und seine Umkehrung (T. 12). Diese Beziehung (mit ihren expliziten Wiederholungen des Motivs und seiner Umkehrung in Takt 27-33) wird der Ausgangspunkt zu allen weiteren Entdeckungen im Werk.

5 Trotzdem waren viele Eingriffe und Erläuterungen notwendig. Das Wort stonikal beispielsweise verwendet Simon in drei verschiedenen Bedeutungen: Manchmal ist damit irgendeine Funktion gemeint (nicht unbedingt die Tonika), manchmal ist der eine Grundton des Stücks damit angesprochen, manchmal ist die Funktion der Tonika insgesamt gemeint. Hier habe ich manchmal im Text korrigiert und in der Fußnote den Ausdruck des Originals angegeben, manchmal auch den Text belassen und in der Fußnote das jeweils Gemeinte klargestellt.

6 Die Unterscheidung von simpliziten den ganzen Text.

7 D.h. der Komposition.

8 Das charakteristische Detail ist ein nur scheinbarer Einfall deswegen, weil es sich als Konsequenz allgemeiner, über die Passage hinaus gültiger Ordnungsprinzipien deuten lässt. 


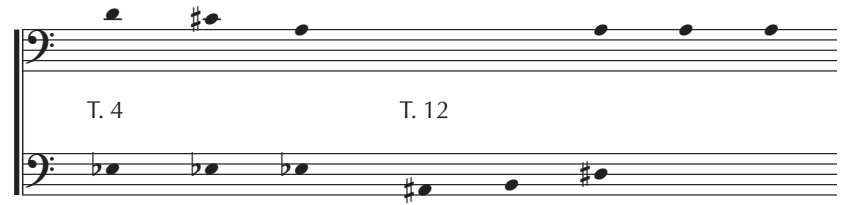

Beispiel 1

Zum repetierenden es erreicht das Anfangsmotiv ein a (T. 4), zur Repetition des a (T. 12) aber ein dis (=es).

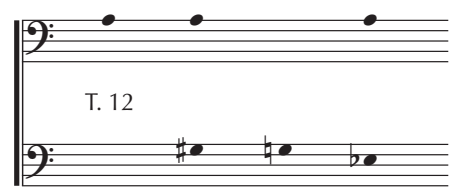

Beispiel 2

Diese sind maximal explizit. Es fehlt noch ein entsprechendes Umkehrungsmotiv für $a{ }^{9}$ Im Takt 17 verborgen, taucht es plötzlich-unerwartet aus einem Gebilde anderer Art als Schluss auf.

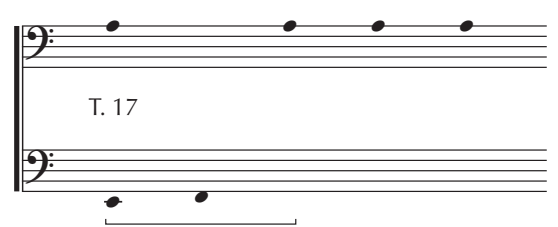

Beispiel 3

NB. Zunächst ist nur die integrierte Anwesenheit der Motive das Ziel unserer Betrachtung. ${ }^{10}-$

9 Zu ais-h-dis gehört als Umkehrung gis-g-es (beide T. 12), beide enden auf demselben Ton dis/es. Es fehlt die auf a endende Umkehrung des Motivs $d$-cis-a (T. 4).

10 D.h. die Anwesenheit einer Gesamtheit von vier aufeinander bezogenen Gestalten des Motivs: $d$ cis-a, e-f-a, ais-h-dis, gis-g-es. 
»Wenn wir diese Motive als Aggregatzustände ${ }^{11}$ betrachten und nach ihren Entsprechungen zusammenfassen, erhalten wir durch die gemeinsamen Töne in der Achse ${ }^{12}$ zwei Quartsegmente. ${ }^{13}$

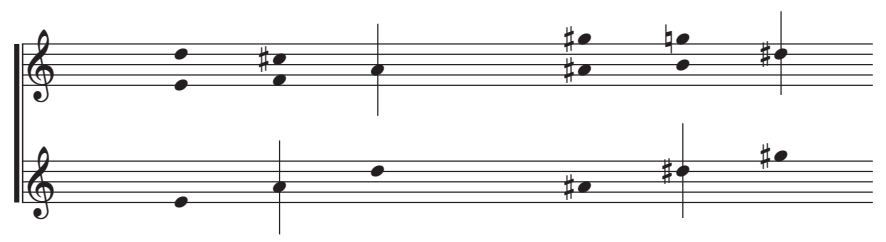

Beispiel 4

Die zwei Achsentöne sind nicht ohne Bedeutung, besonders das a, weil es der Schlusston ist. Wir haben zwei Stellen, welche für diese beiden Töne sprechen: Takt 11 sehr explizit, Takt 23 etwas im Gewebe, aber deutlich. ${ }^{14}$

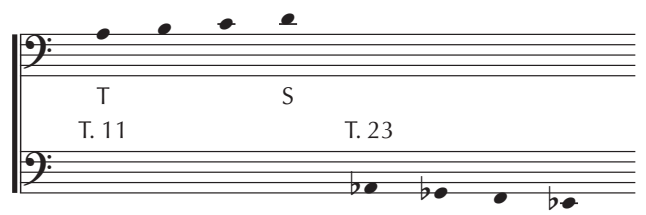

Beispiel 5

Die beiden sind von derselben Struktur, zusammengefasst bilden sie den wohlbekannten Modus aus distanzierten Intervallen, eine Reihe von funktionalen Möglichkeiten. ${ }^{15}$ [...] Wird hier das a als Grundton angenommen, so ergeben sich Subdominant-TonikaBeziehungen.

11 D.h. wenn man von der Reihenfolge der Töne im Motiv absieht.

12 D. h. die gemeinsamen Töne a bzw. dis entscheiden, welche der Motivgestalten zusammen betrachtet werden.

13 Das untere System von Notenbeispiel 4 enthält (gemäß Simons mir ab 1994 mündlich mitgeteilter Terminologie) zwei Tritone: Triton $d$ bis e und Triton gis bis ais.

14 Dies ist der Schluss des ersten bzw. in der Schlussphase des zweiten Teils des Stücks. Die Folge as-ges-f-es steht unmittelbar vor der freien Wiederaufnahme des Anfangsmotivs, die die codaartige Reprise Takt 27 vorbereitet.

15 Im Original findet sich der Ausdruck stonikak statt ,funktionalk. Es geht hier aber allgemein um funktionale Möglichkeiten (in Simons Sinn). Es handelt sich um die alternierende Achtstufigkeit, auch ,Rimsky-Korsakoff-Tonleiter،, zzweiter Modus von Messiaen definiert diese Tonleiter als aus einem generalisierten Funktionsbegriff resultierend, vgl. Haas 2004, 11-19, vgl. auch Lendvai 1995, 8-18 (»Das Achsensystem«) sowie Gárdonyi/Nordhoff, vor allem 156-187. In der vorliegenden Analyse werden häufig die Ausdrücke 'Modus`, 'beschränkter Modus` oder 'Subdominantreihe` und ähnlich für die alternierende Achtstufigkeit verwendet. 


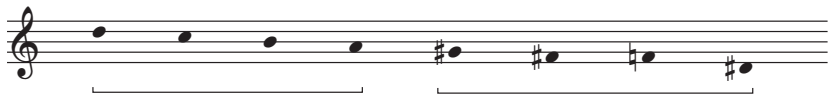

Beispiel 6

NB. In sich genommen ist es eine plagale Tonika. ${ }^{16}$

Wenn es sich so verhält, muss es [...] dementsprechend die weiteren [...] beschränkten Modi auch geben. Wir finden sie auch: in Takt 15-16 ein S-D-Gang. ${ }^{17}$

T. $15 / 16$

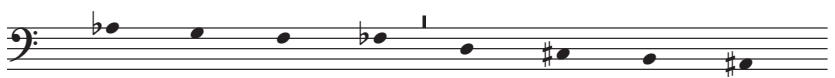

Beispiel 7

Diese Töne sind in einem Gewebe und mit anderen Tönen zusammen, also sozusagen implizit. ${ }^{18}[\ldots]$ Die Stelle ist melodisch interessanter, wirkt [...] chromatisch, da sie durch enge Intervalle zustande kommt, obwohl die Wiederholung des as/gis am Ende eher an die vorige Form des beschränkten S-T-Modus erinnert.

T. 16

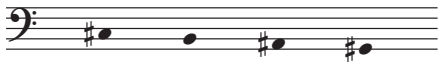

Beispiel 8

Bisher hatten wir also zwei beschränkte Modi: S-T und S-D. Die dritte Gruppe - eben mit der D-T-Tendenz - ist aber nur sehr zerstreut in Form von unterschiedlichen Figuren, teilweise sogar nur in Zusammenklängen da:

16 Sei a Grundton, so sind a, c, es, fis die Grundtöne der Tonika. Fügt man die Quinttöne der Tonika hinzu: e, g, b, cis, so ergibt sich linear g, fis, e, dis (=es), cis, c, b, a. Diese Tonleiter nennt Simon im vorliegenden Zusammenhang sauthentische Tonikar. Fügt man zu den Grundtönen der Tonika die Grundtöne der Subdominante hinzu: $d, f$, gis, $h$, so ergibt sich linear: gis, fis, $f$, es, $d, c, h, a$. Diese Tonleiter, sofern sie auf a bezogen ist (oder lineare Ausschnitte daraus), nennt Simon hier splagale Tonikar. (Dieser Tonvorrat würde sonst als vollständige Subdominante gelten: da nur Grund- und Quinttöne der Subdominante vorkommen, vgl. Haas 2004, 13. Jedoch wird vom Ausdruck iSubdominante der Bezug auf das führende a nicht berücksichtigt.) Von splagaler Tonika ist die Rede, da alle Schritte zur Finalis a plagal sind, falls sie nicht - als Kleinterzschritte oder Vielfache davon - funktional indifferent, also weder authentisch noch plagal sind. Die Tonreihe in Beispiel 6 ist demnach eine plagale Tonika bei Grundton a. Im weiteren Verlauf des Manuskriptes kehrt Simon zu seiner gewöhnlichen Terminologie zurück. Diese speziellen Termini für lineare Funktionen lernte ich erst durch die vorliegende Analyse kennen.

17 S-D-Gang: Tonleiter bestehend aus den Grundtönen der Subdominante $d, f, g i s, h$ und den Grundtönen der Dominante e, $g$, b, cis, zusammen gis, $g$, $f, e, d$, cis, $h$, ais $(=b)$ (Beispiel 7): alle Grundtöne und Quinttöne der Dominante. Der Gang beginnt mit Subdominant-, endet mit Dominantgrundton (S-D).

18 Vgl. Notenbeispiel 24, unterstes System von NB. 


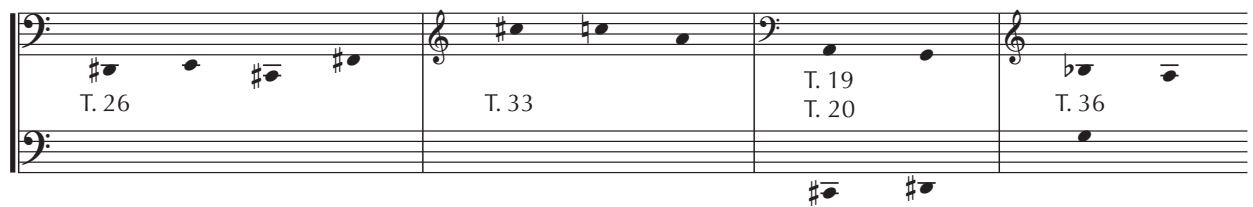

Beispiel 9

1. Takt 26: Ein Segment aus dem Modus, aber mit selbstständiger Motivik [...].

2. Takt 33: Weitere Töne als Teil eines Prozesses.

3. Takt 19-20: Zweitönige Segmente.

4. Takt 36: Der letzte Ton aus der Gruppe (das B) ist eben die zwingendste Dominante für den Schlusston A.

So wird es eine vollständige Tongruppe, die durch das letzte A bestimmt geworden ist.«

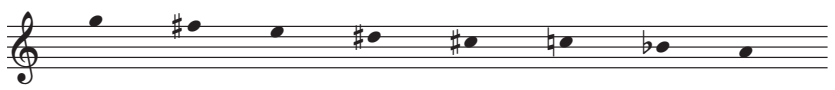

Beispiel 10

„Wir finden einerseits eindeutige Entsprechungen. Takt 5 und 7 wirken jeweils wie kadenzierende Endungen zweier Halbperioden. ${ }^{19}$

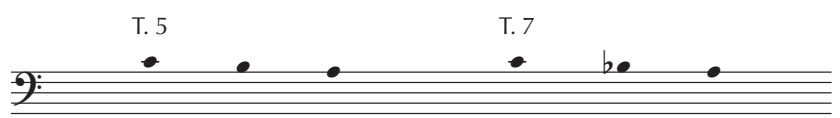

Beispiel 11

Die Zugehörigkeit der übrigen Töne (auch der kleinsten Segmente) können wir unter den drei Modi finden. Die Untersuchung der Coda Takt 33-37 beweist, wie die Reihe der D-T bis zum letzten a auch vertikal wirkt. ${ }^{20}$ [Beispiel 12]

Der überragende Teil der Zusammenklänge repräsentiert die tonikale Gruppe. Später werden wir zu dieser Erscheinung zurückkehren, wenn zwei oder alle drei Modi sowohl linear als auch vertikal gleichzeitig da sind; dies ist die auch von Bartók erwähnte polymodale Chromatik, die einen melodischen (oder harmonischen) Komplex bildet. ${ }^{21}$ [...]

19 Die Folge $c$ - $h$-a drückt eine plagale, die Folge $c-b$-a eine authentische Tonika aus.

20 Damit wird die Reihe der Beispiele für Tonikaklänge von Beispiel 9 vermehrt.

21 Vgl. Notenbeispiel 24. Zu den Begriffen ıbi-modality« und `modal chromaticism` vgl. auch Bartók $1976,376$. 


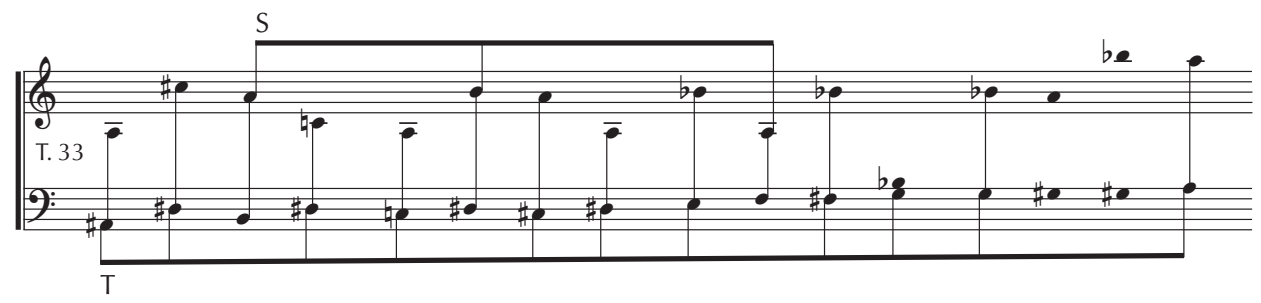

Beispiel 12

Aber von wo gewinnen sie ${ }^{22}$ ihren wirklichen Sinn, so dass sie andere Klänge, die an dieser Stelle (T. 33-Ende) zur Subdominantgruppe gehören, deuten können?

Die Klänge sind mehrdeutig. Mehrdeutig heißt, dass man sie im Sinne eines anderen Organisationssystems verstehen kann.« - „Sie kommen aus einem anderen Aggregatzustand.«-

»Diese Zweideutigkeit stammt aus der Gruppe der Grundfiguren. ${ }^{23}$ [...] Bevor wir zur Gruppe der Grundfiguren ${ }^{24}$ zurückkehren, um das andere Organisationssystem ${ }^{25}$ herauszufinden, untersuchen wir jetzt die Coda, welche von der betonten, ganz besonders akzentuierten Rekapitulation des Kernmotivs und seiner Umkehrung ausgeht.

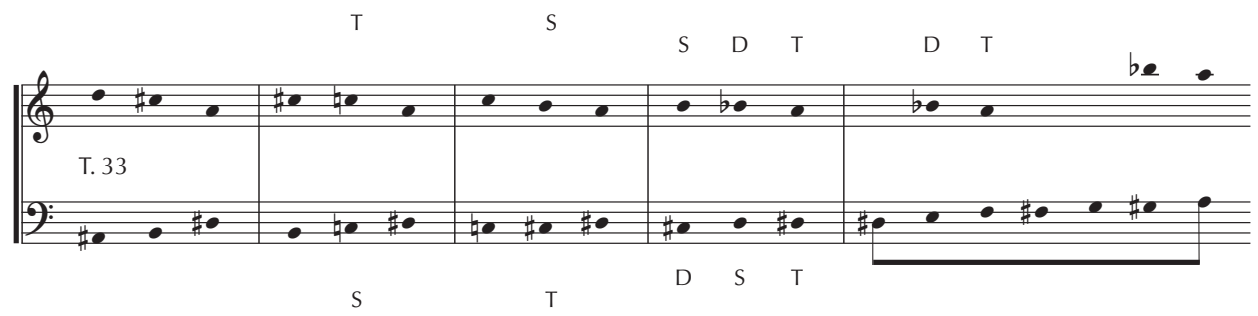

Beispiel 13

Die Grundfigur - das initiierende Motiv - enthält eine große Terz, ihre Tritonustransposition und die Umkehrungen auch dementsprechend. ${ }^{26}$ Aus der Tritonustransposition folgt, dass alle zu derselben Ganztonreihe gehören. Hier ist wichtig, dass diese Ganztonreihe auch die Tonika enthält. ${ }^{27}$

22 Die Zusammenklänge.

23 Gemeint ist das Motiv $d$-cis-a samt seiner Transposition und seiner Umkehrung. Dieses Motiv passt in keine Funktion: $d$ ist Subdominantgrundton, cis ist Dominantgrundton (oder Tonikaquintton), a Tonikagrundton.

24 Original: »Bevor wir zur Grundtongruppe zurückkehren...»

25 Das andere Organisationssystem neben den Funktionen, den drei beschränkten achtstufigen Modi.

26 Zur Terz cis-a des Motivs $d$-cis-a (T. 4) gehört die tritonusentfernte Terz g-es des Motivs gis-g-es (T. 12). Durch die Umkehrungen kommen die Terzen $h$-dis (T. 12) und f-a (T. 17) hinzu.

27 Mit `Tonika ist hier der Grundton des Stücks, a, gemeint. 


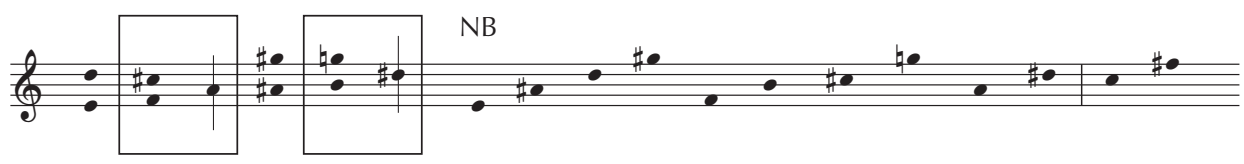

NB
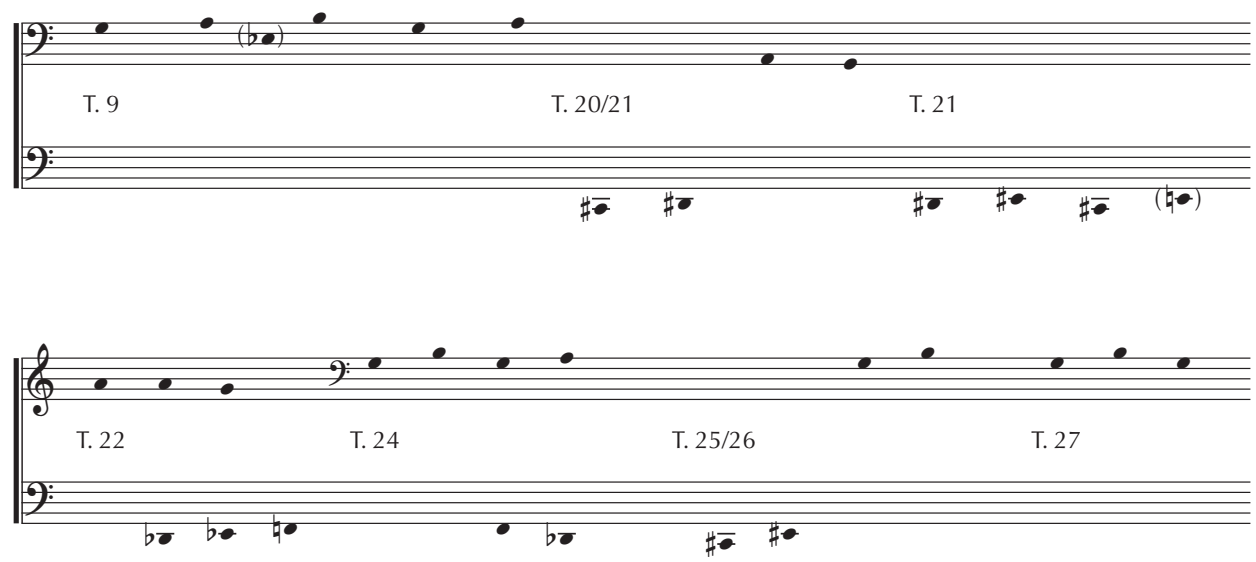

Beispiel 14

NB. Alle zehn Töne sind im Tritonus-Verhältnis durch die a-dis-Grunddisposition. Ein Tritonus fehlt aber: c-fis.

Die Ganztonreihe der Tonika ${ }^{28}$ ist variabel ins Spiel gebracht. Betrachten wir einige reine Ganztonstellen. Dabei sehen wir jetzt ab von den Takten 4, 12, 13 und auch von Takt 17, wo die Ganztönigkeit thematisch ist. ${ }^{29}$

Zu NB unter Beispiel 14:

1. Takt 9: Ganztöne um den Grundton a.

2. Takt 20-21: Ganztöne als Teil des Tonika-Modus. ${ }^{30}$

3. Takt 21: Weitere Ganztöne. ${ }^{31}$ Zweideutig: die Töne $a$, dis, $f$ sind auch Subdominan$t^{32}$, aber mit den nächsten Tönen wird zur Tonika korrigiert.

28 D.h. die Ganztonreihe mit a. - Die Ganztonreihe ist also das andere Strukturprinzip neben den Funktionen.

29 D. h. wo die Ganztönigkeit vom Grundmotiv gegeben ist.

30 Die Töne cis, dis, a, g in Takt 20/21 lassen sich als Schnittmenge der Tonika (aus Grund- und Quinttönen) mit der Ganztonreihe auf $A$ bestimmen.

31 Zu cis, dis, $g$, a kommt $f$ als fünfter Ton der Ganztonreihe hinzu.

32 Die ersten drei Töne von Takt 21, a-dis-f, gehören der Subdominante und der Ganztonreihe auf a an. Die vollständige Schnittmenge der Subdominante mit der Ganztonreihe auf a wäre a-h-dis-f. 
4. Takt 22: Ganztöne. (Den wirklichen Sinn siehe später ${ }^{33}$ ).

5. Takt 24: Variierte Rekapitulation von Takt 9. Dazu dieselbe Terz noch einmal, tritonusversetzt. ${ }^{34}$

6. Takt 25-26: Ein doppeldeutiges Spiel [...], aber die ‘ganztönigen großen Terzen sind eindeutig. ${ }^{35}$

7. Takt 27: , Resumee`.

Wie vorher bei den Tritoni erwähnt, fehlte nur einer, c-fis. Wir können die zehn Töne auch als Quartreihen auffassen [...], so dass sich zwei Quartreihen in Tritonusabstand ergeben. ${ }^{36}$ (Siehe Beispiel 4.)
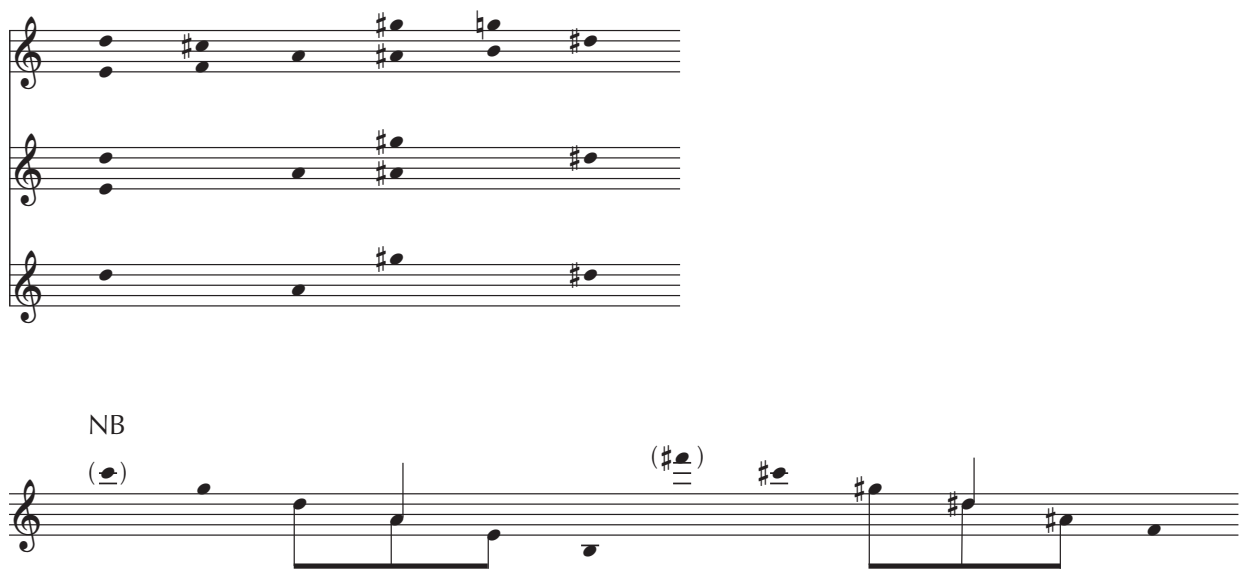

Beispiel 15

Die zwei Quartreihen haben keine Verbindung.

Aber so erhalten wir eine plagale Tonika. ${ }^{37}$ Eine authentische Tonika wäre folgendermaßen.

33 Vgl. Notenbeispiel 18 und 26 mit Kommentar.

34 T. 24: $g$ - $h$-g-(a) sowie $f$-des-f.

35 Es handelt sich um die Schnittmenge der Dominante mit der Ganztonreihe auf a.

36 Notenbeispiel 15 zeigt unter NB zwei Pentatone: eins von $g$ bis $h$, eins von cis bis eis ( $f$ ). Dabei bildet das mittlere Triton jeweils das Rahmenintervall des Ausgangsmotivs und seiner Umkehrung ( $\underline{D}$-Cis$\underline{A}$ sowie $\underline{E}-F-\underline{A})$. Die beiden äußeren Töne des Pentatons ergeben den mittleren Ton des jeweils anderen Motivpaars, z. B. Gis- $\underline{G}-E s$ und Ais- $\underline{H}$-Dis.

37 Der Ausdruck splagale Tonika bezieht sich auf die Folge $d$-a, gis-dis (Notenbeispiel 15, 3. Zeile), vgl. dazu Anmerkung 16. 


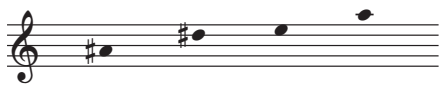

Beispiel 16

Damit die Tonika-Gruppe in solcher Strukturierung vollständig sein könne, sind außer diesen noch die folgenden Töne nötig.

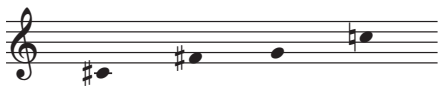

Beispiel 17

Die Töne von Beispiel 16 sind nur fiktiv, in verschiedensten Hinweisen da ${ }^{38}$, aber die von Beispiel 17 werden vielseitig vorbereitet mit großer Energie gebracht. Nach der Imitation $^{39}$ noch ein Tonika-Segment. ${ }^{40}$
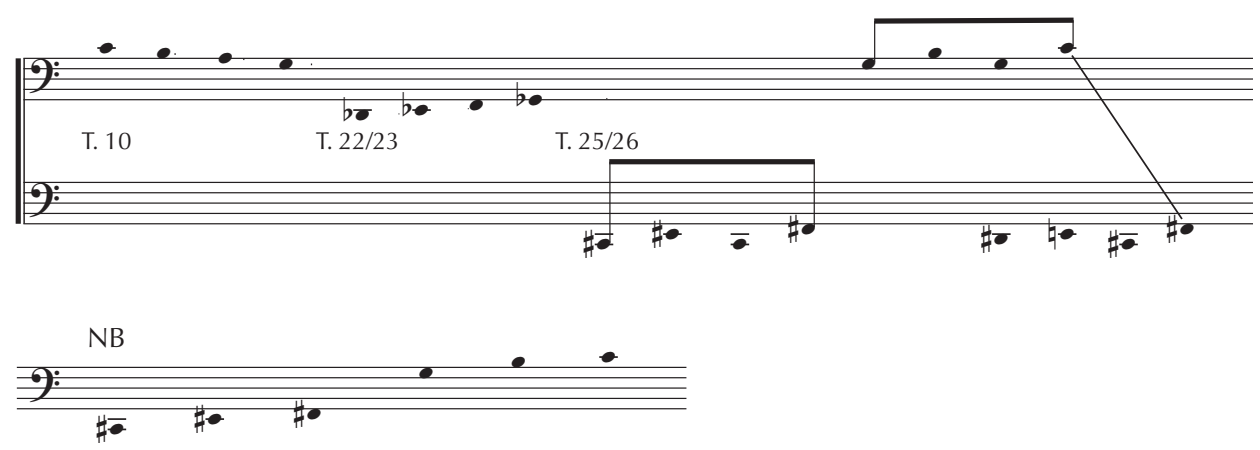

Beispiel 18

NB. Gleichzeitig geschieht die Vervollständigung dieser Art Struktur der tonikal gerichteten Gruppe und die der fehlenden zwei Töne zum Tonvorrat der Tongruppe des Motivs. ${ }^{41}$

Nebenbei ist das erreichte rein imitierte Motiv ein freier Krebs des Grundmotivs ${ }^{42}$ und so die Rekapitulation der Grundidee.«-

38 Diese Töne einer authentische Tonika sind in den Motive ais-h-dis (T. 11/12) und e-f-a (T. 17) vorhanden, sofern man nur ihre Ecktöne (ais, dis, e, a) betrachtet, vgl. auch Notenbeispiel 15, 2. System, Unterstimme.

39 Mit >Imitation` ist die Folge g-h-g-C (T. 25/26 r. H.) im Verhältnis zu cis-eis-cis-fis (T. 25 I. H.) gemeint.

40 Beim `Tonika-Segment handelt es sich um die Folge dis-e-cis-fis (T. 26 I. H.), vgl. Notenbeispiel 9/1.

41 Es werden fis (T. 25 I. H.) und c (T. 26 r. H.) angesteuert. Diese beiden Töne fehlten der Entfaltung des Anfangsmotivs $d$-cis-a zum Zwölftonraum, vgl. Notenbeispiele 14 und 15. Andererseits entsprechen die Haupttöne cis, fis, $g$, c der authentischen Tonika von Notenbeispiel 17.

42 cis-eis-fis wäre der genaue Krebs (transponiert) des Anfangsmotivs. 
»Der kleine Schlussteil des periodischen Ablaufs ab Takt $15^{43}$ kann in sich bimodal gewertet werden, und zwar als Subdominante und Tonika. Was das Ganze bedeutet, ist klar, weil alles sich auf $A$ bezieht. Eine Erscheinung klar aufweisen kann man [...] nur abgesehen von den ordnenden Kräften der anderen. ${ }^{44}$

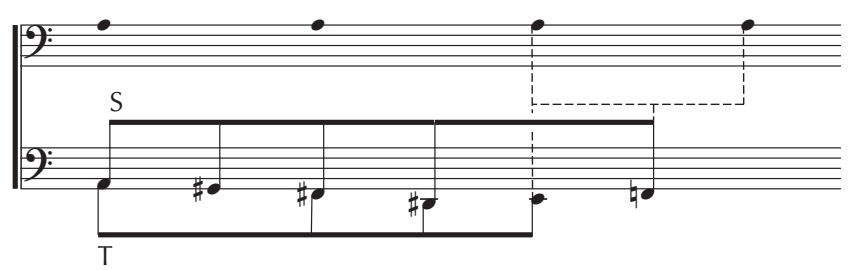

Beispiel 19

»Takt 21: eine nachfolgende mikro-bimodale Stelle (verwandt mit Beispiel 19).

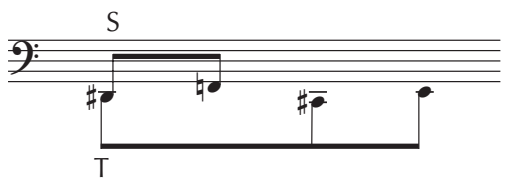

Beispiel 20

Takt 26: Hingegen sind die letzten vier Töne eine reine Äußerung der Tonikagruppe.

$$
\text { T. } 26
$$

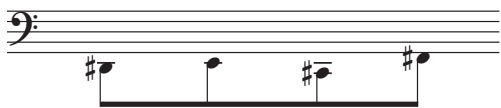

Beispiel 21

Um die bi- oder polymodalen Zustände gut zu begreifen, müssen wir zuerst die Grundposition in Acht nehmen, dann die [...] Möglichkeiten im gegebenen Raum untersuchen. Vom Anfang bis Takt 7 enthalten (abgesehen von dem repetierenden Ton) die fast periodisierenden melodischen Bewegungen alle Töne zwischen $d$ und a. In diesem Intervallraum gelten die folgenden Modusmöglichkeiten:

43 Was in Takt 15 beginnt, endet in Takt 17.

44 D.h. sobald man sich auf die Wahrnehmung einer Funktion konzentriert, gerät die andere ins sperzeptive Hintertreffen«, in diesem Zusammenhang des Öfteren Simons Rede von »Zwei-« und »Mehrdeutigkeit«. 


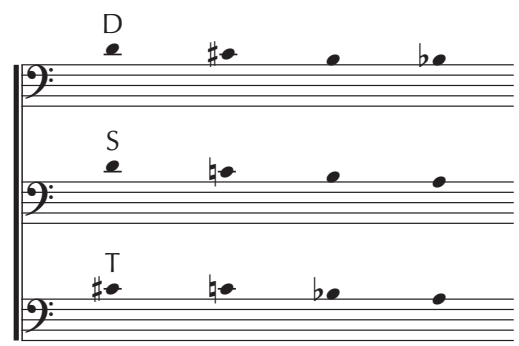

Beispiel 22

Es ist dichte Chromatik vorhanden, die von der Grundfigur ausgeht. In sich bimodal (S, T) mit zwei gemeinsamen Tönen (a-es). ${ }^{45}$ Dazwischen stehen die Endungen: reine Subdominante (T. 5), reine Tonika (T. 7). In Takt 11 die viertönige Tonreihe gehört vollständig zur Subdominante. ${ }^{46}$

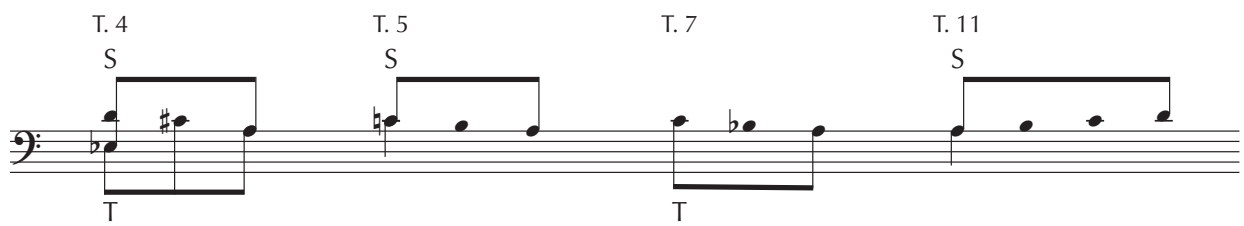

Beispiel 23

Es hat sich schon an einigen Stellen gezeigt (sowohl vertikal als auch linear), dass ais mit $a$ oder $b$ mit a tonikale Klänge sind; dann muss a mit gis Subdominante sein, die drei Töne gis, $a$, $b$ bilden den Schluss. Ab Takt 12 bis 14 [Beispiel 24] besteht eine Zweideutigkeit wegen dem aus den Figuren sich ergebenden Ganztonsinn in Bezug zu a. Diese sind aber aus dem modalen Sinn empfangen ${ }^{47}$, sie sind auch in den (über unterschiedliche funktionale Richtungstendenzen verfügenden) Modi sinnvoll: Polymodalität.

NB. In Takt 13 wird das Großterzgerüst hervorgehoben durch eine Elision in der Abfolge der Figuren. Regelmäßig geführt nämlich würde es ein zwölftöniger Gang sein. ${ }^{48}$ (Dort wird ein Ton wiederholt, eben es [dis], dazu das repetierende a.) Regelmäßig geführt wäre es-e-gis die entsprechende Antwort für gis-g-es. ${ }^{49}$ Aber das Wahrschein-

45 Die Grundfigur passt in keine Funktion, da sie zwei Funktionen enthält: Subdominante (D) und Tonika (cis = Quintton, $a=$ Grundton), vgl. Anmerkung 23. Beispiel 23 zeigt, dass die Töne $a$ und es sowohl der Tonika (als Grundtöne) als auch der Subdominante (als Quinttöne) angehören.

46 Diese Tonfolge wurde oben als plagale Tonika bezeichnet (vgl. Beispiel 6). Der Tonvorrat der plagalen Tonika ist der der Subdominante. - Es zeigt sich, dass an dieser Stelle die Dominante, deren Gestalt innerhalb der Quarte $A-D$ in Beispiel 22 angegeben wurde, nicht vorkommt.

47 D. h. aus den Funktionen.

48 Diese regelmäßige Führung ist im 3. System von NB zu Beispiel 24 dargestellt.

49 Dann würde das hypothetische es-e-gis (T. 13/14) auf ais-h-dis (T. 13 mit Auftakt) in ähnlicher Weise antworten, wie gis-g-es (T. 12) auf ais-h-dis (T. 12 mit Auftakt) antwortet. 

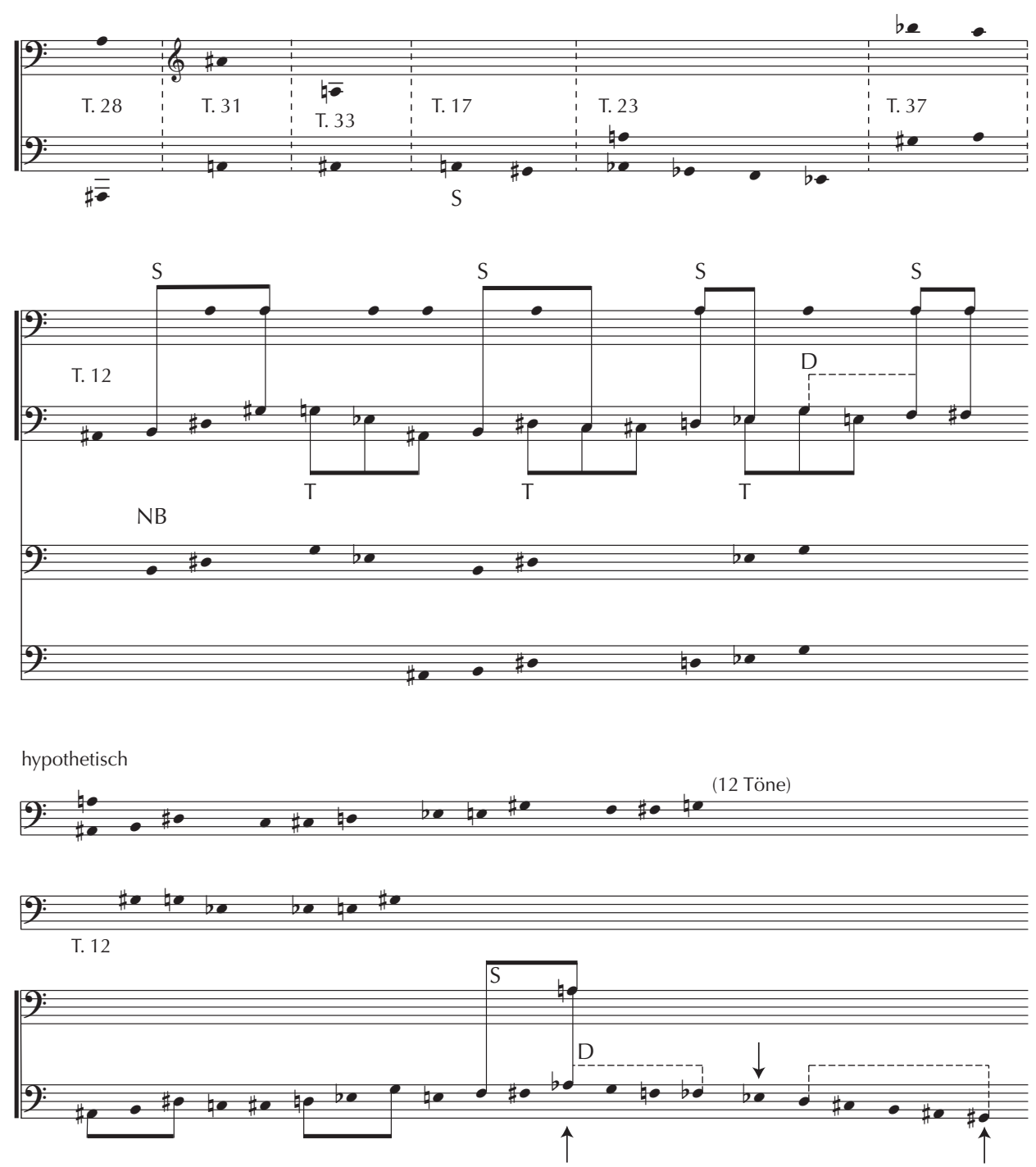

Beispiel 24

lichste ist, dass in der tatsächlichen Version die mit es beginnende Figur (T. 13, 4. Viertel) subdominantisch endet (es-f-fis, vgl. Beispiel 24) und die Subdominante in Takt 15 mit dem vorher ausgebliebenen zwölften Ton as vervollständigt wird ${ }^{50}$; das a/as ist betont.

50 Diese Subdominante beginnt in Takt 13 mit $h$-c-d plus Orgelpunkt a, vgl. Notenbeispiel 24, 2. System (Darstellung zu T. 12 ff.). 
Zugleich beginnt mit as ein Dominantgang ${ }^{51}$, der fis zugunsten von $g$ elidiert. ${ }^{52}$ Das in die Mitte des Dominantgangs gestellte es [...] erhält a-es aufrecht. Der letzte Ton des Dominantgangs gis gibt das vorige as zurück.« -

"Zu Takt 24-26: Die drei Hauptrichtungen der Zusammenhänge in der oberen Stimme sind folgende:

a) Ganztonreihe des Grundtons - weitere Töne in der zweiten Stimme.

b) Die tonikale Gruppe - die weiteren Töne im nächsten Takt, in der zweiten Stimme.

c) Die Grundfigur in einem transformierten $\mathrm{Krebs}^{53}$ - sie ist schon eine Imitation - Wiederholung der mehrmaligen Ansätze der vorangehenden Tritonusparallele.

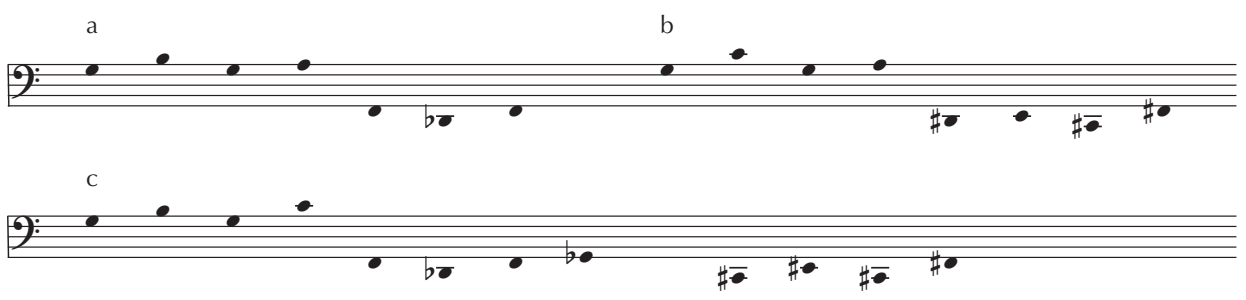

Beispiel 25

Zusammenfassende Bemerkung zu den Beispielen 5, 15, 16, 17, 18.

- Beispiel 18: Das Gerüst/die Rahmentöne sind aus der Tonikagruppe.

- Beispiel 5: Das Gerüst ist aus der Subdominantgruppe. Der Tonvorrat enthält den ganzen Subdominantmodus.

- Takt 22-23: Die erste Hälfte ist zusammengewoben mit der Tonreihe des Tonikagerüstes $^{54}$ und gelangt zum transformierten Krebs der Grundfigur. Der zweite Teil der Tonreihe stellt sich dar in der Fortführung der Oberstimme.

Die einander entsprechenden Teile sind in Umkehrungen dargestellt. Es wird auch klar, dass die gemeinsamen Töne aus der Subdominantreihe sind. Solche Deutungen scheinbarer Dur-moll-Tonleitersegmente sind sehr häufig bei Bartók.

51 Vgl. Notenbeispiele 7 und 8.

52 Vgl. Notenbeispiel 24, NB, unterstes System: as-g-f-fes gegenüber e-f-fis-as.

53 Vgl. Notenbeispiel 18.

54 Die Tonreihe besteht aus dem subdominantischen Tetrachord as-ges-f-es und der diesen Tönen entnommenen Ausfüllung der tonikalen Quarte des-ges. 

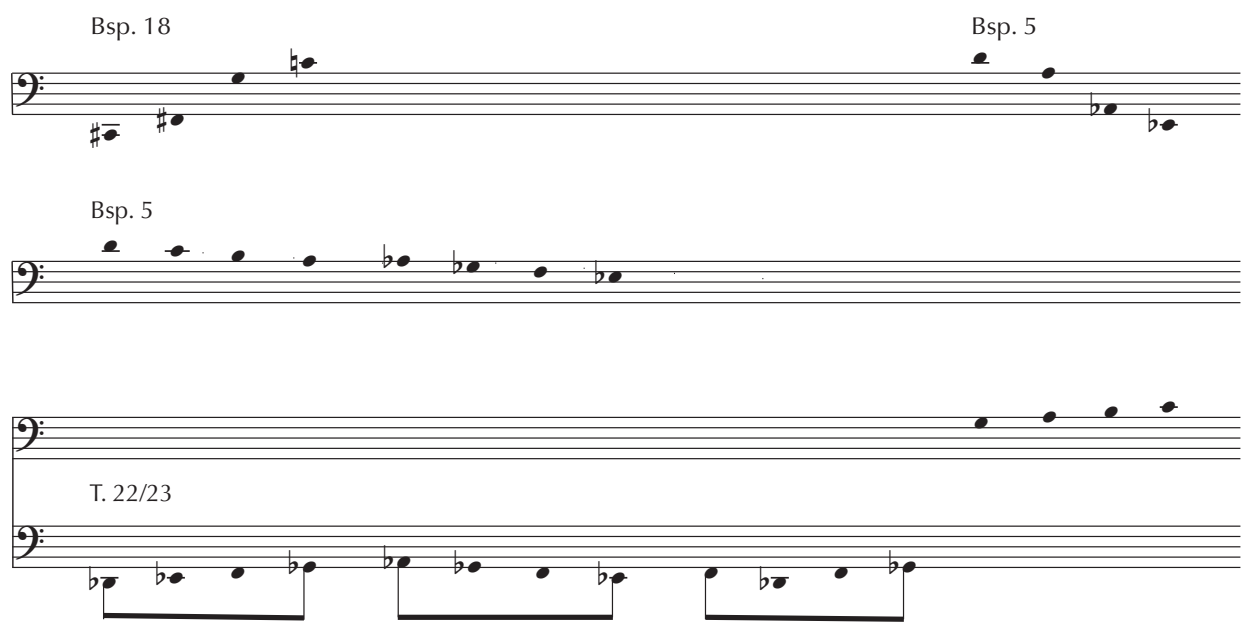

Beispiel 26

Die Tonikareihe wäre so: NB.«-

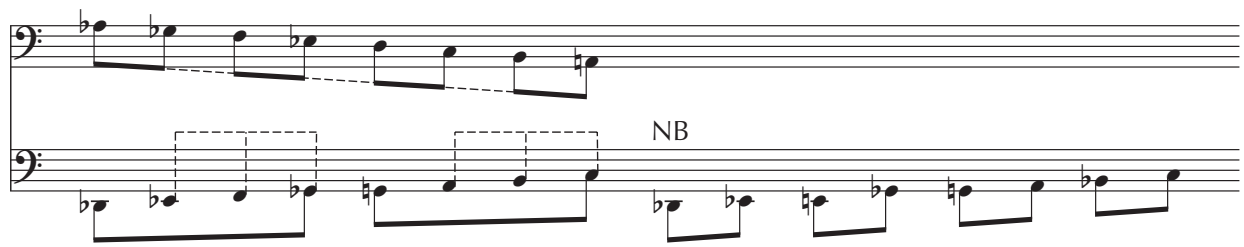

Beispiel 27

»Bemerkung zum Beispiel 12:

Im Licht der Wichtigkeit der tonikalen Ganztongruppe (d. h. der Ganztonreihe, die den Grundton A enthält) ist die Zweideutigkeit in der Coda infolge des Klangnacheinanders noch prägnanter darzustellen. Aber beide dienen demselben Zweck, das A zum höchsten rresonanten` Generierungspunkt zu bringen. «5 $^{55}$

»In diesem Sinne ist a die Tonika und das Stück ist überhaupt tonal. Diese durch und durch organisierte Komplexität des Komponierens benennt Bartók in diesem Sinne mit einem einzigen Ton als Grundton: in a.»

55 Unter »beide« sind hier einerseits die Funktionen Tonika und Subdominante sowie andererseits die Ganztonreihe gemeint. 


\section{Geteilte Arpeggien (Mikrokosmos Nr. 143)}

»Dieses Stück kann man, wie viele andere im Mikrokosmos, als eine Kompositionsübung, -lehre oder wie ein Rätselstück auffassen, das - systematische Ordnungsprinzipien betreffend - eine Vielfalt von Schnittpunkten demonstriert, aber in einem wirklich schönen Musikstück.

Das Rätsel fängt an mit zwei Ganztonschritten, zusammengebunden mit einer Quart.

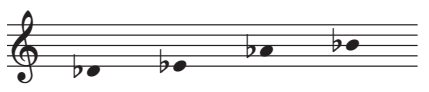

Beispiel 28

Es berührt im Laufe gehäufter Imitationen verschiedene Intervalle, bis es einen fünften neuen Ton erreicht, der ins Zentrum gestellt mit zwei Tönen aus den vorigen Vieren einen melodischen Kern bildet [...].

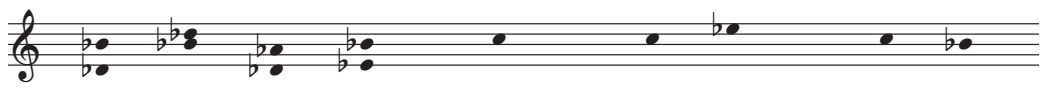

Beispiel 29

Die bisherigen fünf Töne bilden ein Pentachord.

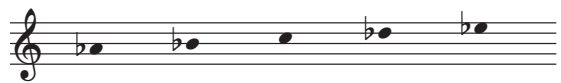

Beispiel 30

Diese einleitenden Töne verweisen unmittelbar auf das nun folgende Arpeggio, es sind seine beginnenden und schließenden Töne.

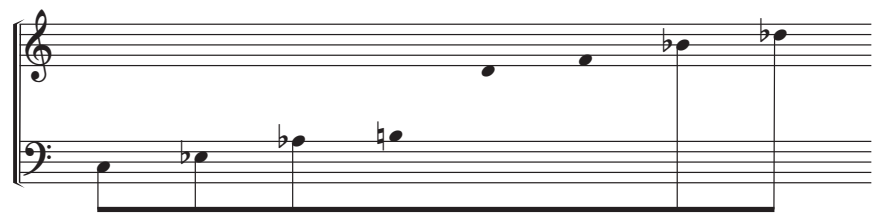

Beispiel 31

Dieses erste Arpeggio wird zweimal transponiert wiederholt. 

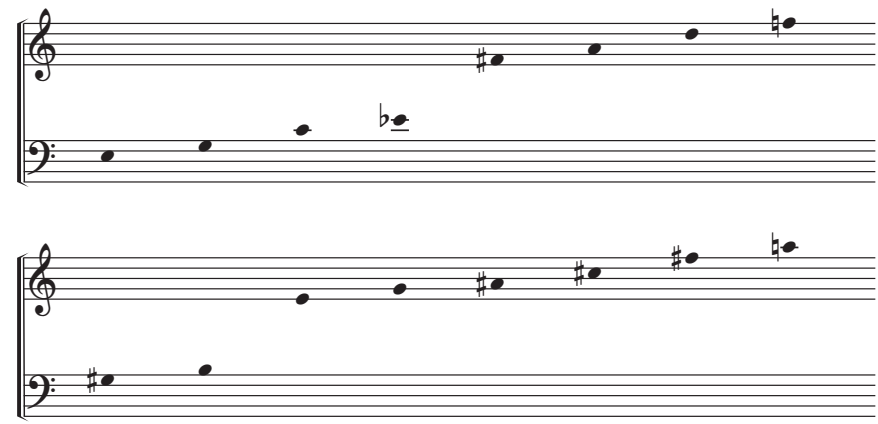

Beispiel 32

Wenn wir mit den zwei schließenden Tönen des dritten Arpeggio beginnen würden, dann hätten wir zweimal zwölf Töne in einer Art Perpetuum mobile von Kleinterz-Gruppen [...].

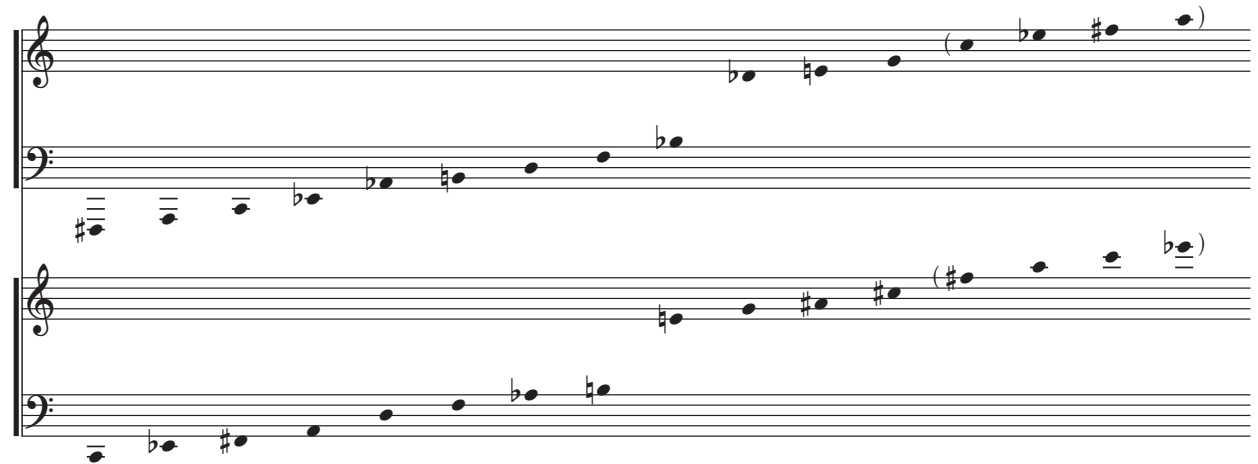

Beispiel 33

Warum die 24 Töne nötig sind, zeigt sich erst, wenn wir sämtliche Quartschritte in eine Gruppe sammeln. ${ }^{56}$

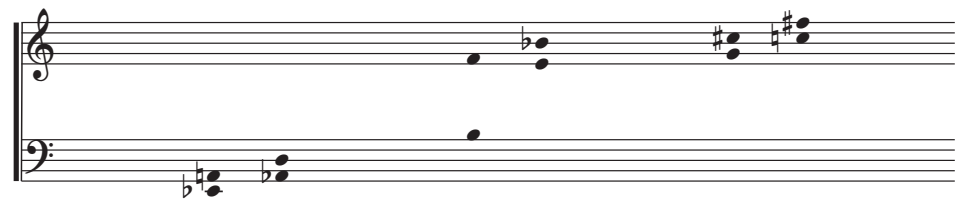

Beispiel 34

56 Erst nach 24 Tönen nämlich ergeben sich sechs Quarten, die alle zwölf Töne umfassen. Nach zwölf (bzw. dreizehn) Arpeggientönen (Beispiel 33, oberes System) hat man erst drei Quarten mit insgesamt 6 Tönen. In Beispiel 34 wurde die Folge $f^{0}-b^{0}$ irrtümlich eine Oktave zu hoch geschrieben (vgl. Beispiel 33). 
Nun in der Reihenfolge, wie sie im Stück erscheinen:

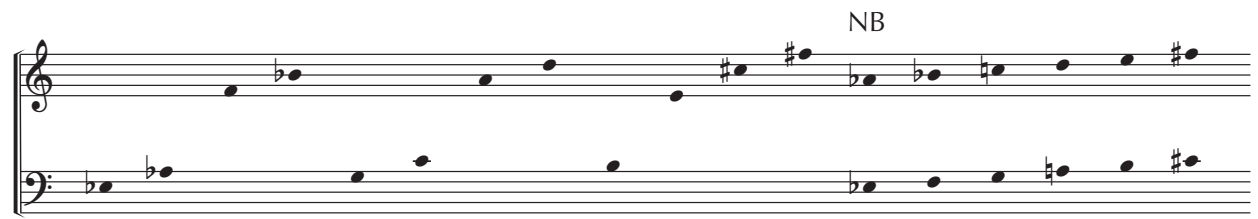

Beispiel 35

NB. Wir treffen auf zwei Ganztonreihen, und im Werk zeigt sich sofort der letzte Ton als Ausgangspunkt für die weiteren Arpeggien.

Dann fis-e-d-c, darum später fis-e. ${ }^{57}$

Die entstandenen zwei Ganztonreihen in Quartabstand enthalten zwölf Töne. Die sonderbare Geschlossenheit aber dieser 24 Töne der drei Arpeggien zeigen zweimal zwölf Töne von anderer Herkunft und [...] anderem Sinn der Integration. ${ }^{58}$

12

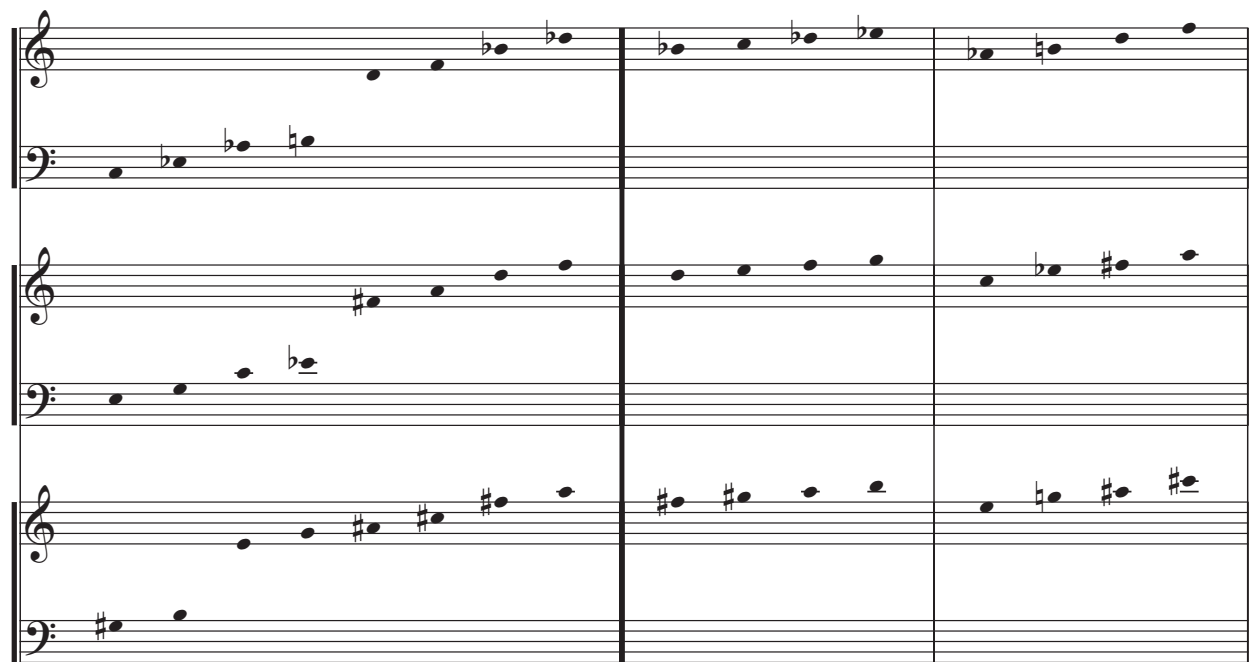

Beispiel 36

57 Vgl. dazu Beispiel 39, unterstes System (NB). fis-e-d-c wären demnach die führenden Töne ab Takt 11: fis (T. 11), e (T. 12), d (T. 12, 4. Viertel), c (T. 13, 2. Viertel r. H.). Mit »fis-e« sind wahrscheinlich die symmetrisch zueinander stehenden Töne fis ${ }^{1}$ und $e^{2}$ in den Takten 18-25 gemeint. Das Wort »darum « deutet vielleicht an, dass die Töne fis-e als fis-e- $d$-c, verkürzt um die beiden letzten Töne, aufzufassen sind.

58 In der Spalte 2 von Beispiel 36 finden sich jeweils alle Grundtöne von Subdominante, Tonika, Dominante. Es handelt sich dabei jeweils um die mittleren vier Töne des in der linken Spalte stehenden 
Die nächste Aufteilung zeigt, wo die Rolle der gerüstbildenden großen Terzen hervortritt. Dementsprechend das andere Ganztongerüst (mit den anderen analogen 24 Tönen $^{59}$ ):

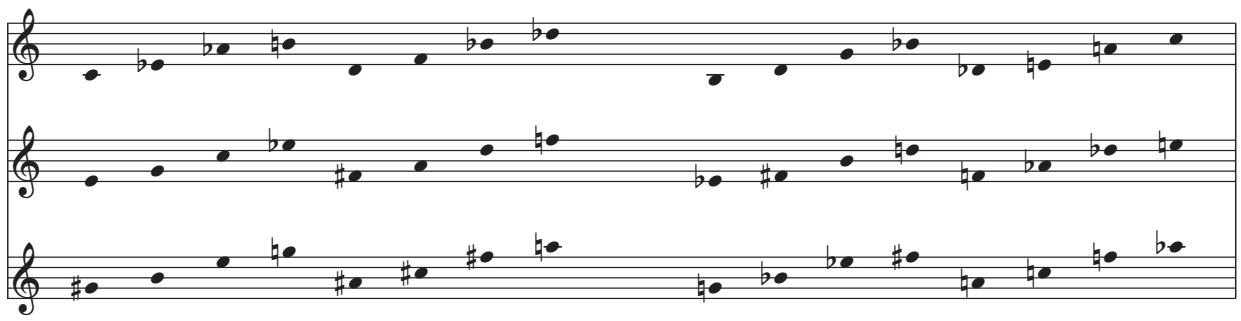

Beispiel 37

NB. Dieses Beispiel veranschaulicht auch die symmetrische Teilung der Arpeggien, die [...] die kadenzartigen Bestätigungen der funktionalen Richtung, auf bestimmte Punkte gerichtet, anstreben, so in Takt $14-22$, Takt $57-63^{60}$ und bis zum letzten einzelnen $c$ in Takt 68-80. ${ }^{61}$ In diesem Beispiel sind alle zwölf gezeigt. [...]

Und schließlich aus dem vertikalen Bild sich ergebende zwölftönige Überquerungen ${ }^{62}$ :

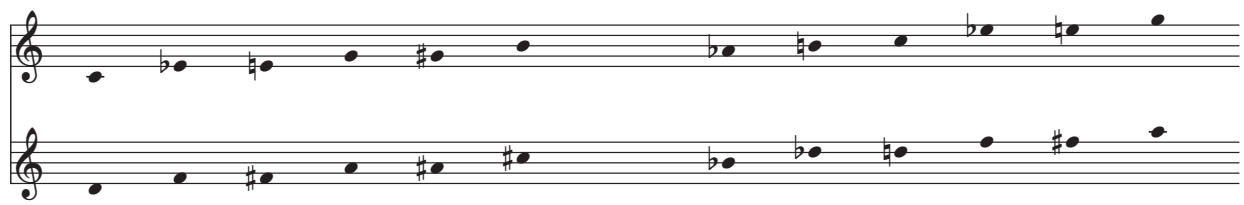

Beispiel 38

Arpeggios. Unter der Spalte 1 von Beispiel 36 sind jeweils die ersten und die letzten beiden Töne des links notierten Arpeggios zusammengefasst. Es handelt sich jeweils um Schnittmengen aus einem Hexaton und einer Funktion, z. B. im oberen System Hexaton des bis $c$ und Tonika etc. Auch die Tetrachorde der Spalte 1 enthalten zusammen alle zwölf Töne.

59 Die drei Systeme von Beispiel 37 stehen in Großterzabstand. Das »andere Ganztongerüst mit den anderen analogen 24 Tönen« besteht in den Takten 50-56 (darauf bezieht sich die rechte Hälfte von Beispiel 37).

60 Im Originaltyposkript steht 62 statt 63. Da Takt 63 aber analog ist zu Takt 22, scheint es mir, als sei in beiden Fällen der erste Klang von Takt 22 bzw. Takt 63 mitgemeint, nicht aber der Schluss dieser Takte.

61 In den Takten 14-22 sowie 57-63 werden jeweils zwei viertönige Arpeggien derselben Funktion verknüpft, in Takt 14-22 der Tonika, in Takt 57-63 der Subdominante, daher der Ausdruck »funktionale Richtung (im Original steht »tonikale Richtung«, vgl. dazu Anmerkung 15). Dagegen enthält jedes zusammengesetzte achttönige Arpeggio der Takte 6-13 und 50-56 Töne verschiedener Funktionen (vgl. Beispiel 36).

62 Das obere System von Notenbeispiel 38 enthält die ersten beiden Töne der linken Spalte von Beispiel 37, bzw. die ersten beiden Töne der Takte 6, 8, 10; darauf folgt das zweite Tonpaar der linken Spalte von Beispiel 37 etc. Es handelt sich um die wohlbekannten Konstrukte: Ila im oberen, Ilb im unteren System. Eine analoge Darstellung für die Takte 50-56 ergäbe die Konstrukte la und lb (zu dieser Terminologie vgl. Haas 2004, 27-29). 
NB. In Takt 30-43 werden die tonalen Beziehungen durch Strukturen solcher Art bzw. aus ihnen weitergeführten Strukturen repräsentiert. ${ }^{63}$

$c$ ist als Schlusston deklariert. Wir können in unserem breiten Sinne Tonika sagen. Dieser breite Sinn kommt aus den drei beschränkten Modi, welche in gleicher Weise und Zahl je vier gleich berechtigte Tonikamöglichkeiten enthalten. ${ }^{64}$

Wenn eine der drei Reihen/Tongruppen den letzten Grundton enthält, so ist sie die Tonikagruppe, dementsprechend eine andere die Subdominantgruppe und die dritte die Dominantgruppe.

Die Tongruppen - mindestens bei diesem Stück - sind als Felder der Funktionsrichtungen für das ganze Stück gültig."« ${ }^{65}$

»Nun die Analyse weiter. Die Arpeggien veranschaulichen die linearen Übergänge durch ihre gemeinsamen Töne zwischen den funktionalen Tonfeldern. ${ }^{66}$ Die abgekürzte Abfolge der Arpeggien zeigt ein harmonisches Nacheinander der Übergänge. ${ }^{67}$

T. 11

S

$\mathrm{D}$

$\mathrm{T}$

S

$\mathrm{D}$

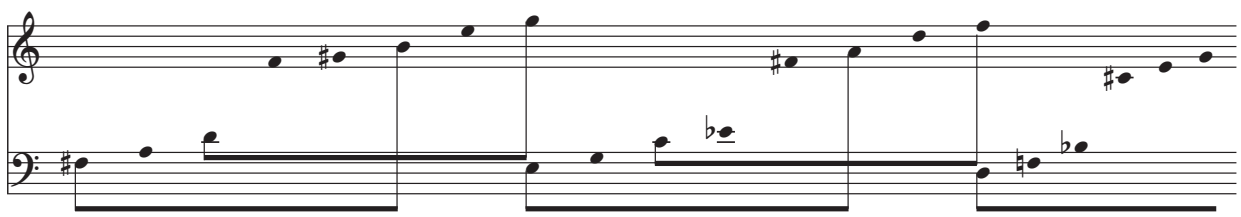

NB
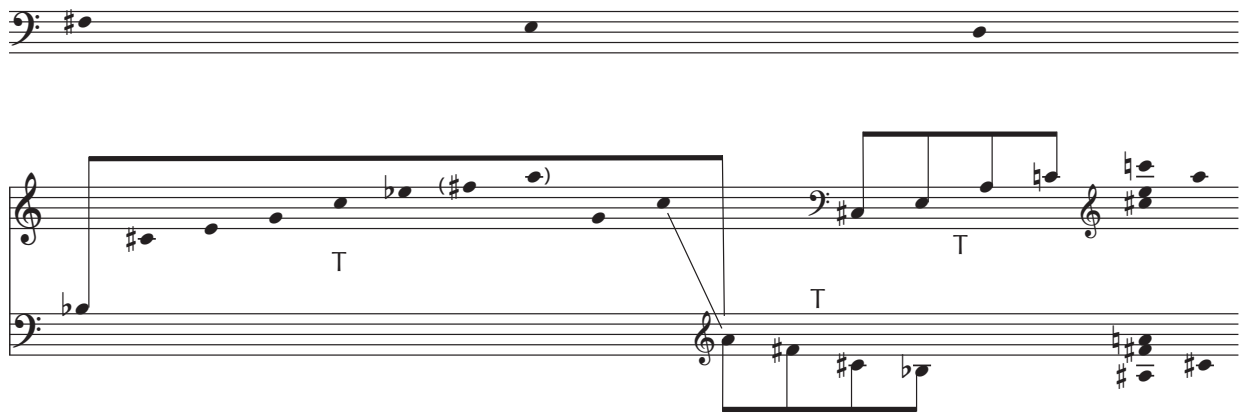

Beispiel 39

63 Auf diesen Gedanken kommt der Text nur in einer winzigen Andeutung zurück (vgl. Beispiel 44). Gemeint sind vielleicht die Konstrukte $a$-fis-d, f-des-b (T. 30, 32, Ilb), g-e- $h$, es-as-c (T. 35/36, Ila) sowie ges-eses-ces, es-g-b (T. 39-43, Ib). (Im Originaltyposkript lautet die Angabe »T. 30-40« statt »-43«.)

64 Die drei beschränkten Modi sind die drei Modi alternierender Achtstufigkeit, Tonika, Subdominante, Dominante, vgl. Haas 2004, 11-19 sowie Anmerkung 15.

65 D. h. es gibt keine Modulation auf der Ebene der Funktionen (Funktionsrichtungen). Die Reihe/Tongruppe, das Tonfeld, der Modus $c$, des, es, e, fis, $g$, a, b ist in diesem Stück immer Tonika etc.

66 Im Original: »tonikalen Funktionsfeldern«.

67 Die verbindenden Linien in Beispiel 39, die jeweils Dursextakkord, Kleinterzklang, Mollquartsextakkord herausstellen (indem sie sie begrenzen), ergänzen sich in Takt 11/12 zu einem Oktoton $f$ bis fis: fis, $d, h, g$, e, $c, a, f$. Warum die Quarten $d$-g (T. 12/13) und b-es (T. 13) nicht analog herausgegriffen 
NB. Die beginnenden Töne der Arpeggien [sind] eine Ganztonreihe, woran bald die Zusammenklänge ssich erinnern werden a ab Takt $22 .{ }^{68}$

Die zwei Arpeggien ${ }^{69}$ sind acht Töne, aber wegen der gemeinsamen cis, a sind sie sechs. Sie bilden `Zusammenklänge`, und auf solche Weise durchleuchtet analysieren sie ihre Einzelintervalle. Die sechs der tonikalen Gruppe ergänzen sich zum Total, also werden es acht Töne, durch die strukturelle Spiegelbewegung des erwähnten Wechselspiels der Ganztonreihen. ${ }^{70}$ Am Schluss bilden die bisher als Achse fungierenden Töne einen tonikalen Klang.

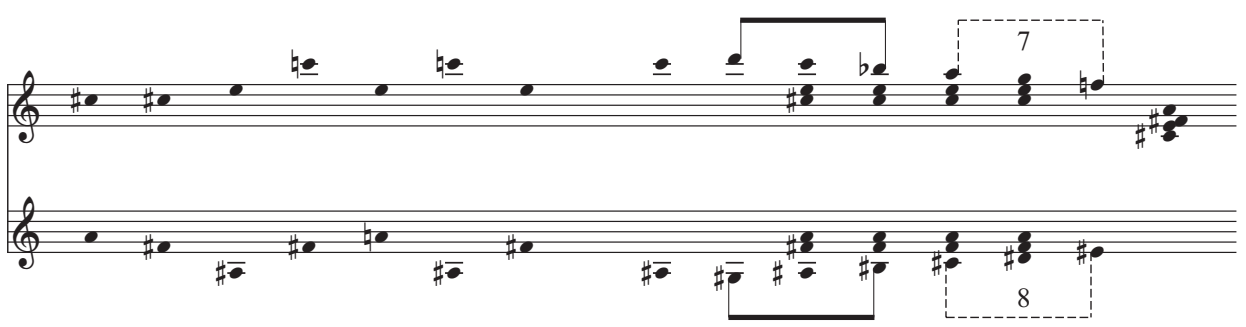

Beispiel 40

[...] Weitere Entfaltung des ersten melodischen Kerns in zwei Richtungen von a ausgehend: $a-d$-a-gis und a-e-a-g. ${ }^{71}$

Zu Takt 30:

Kleinterzgruppen können in regelmäßiger Struktur mittels Quarten zusammengefügt werden ${ }^{72}$, sie können aber auch mittels großer Terzen in tonale Beziehung gebracht werden. [...] Und auch kleine Sekunden können zur Verbindung von beliebig großen Kleinterzgruppen verwendet werden (siehe Beispiel 38), auch große Sekunden sind fähig, Bindeglied zu sein. ${ }^{73}$ Die Kleinterzgruppen - verbunden miteinander durch Quarte, kleine Sekunde, große Terz, große Sekunde - können auch in beliebigem Maße größer oder kleiner sein, also mehr oder weniger in jeder Gruppe.

werden, ist nicht unmittelbar einsichtig. Vielleicht liegt es daran, dass Takt 13 als Ganzes eine Funktion, die Tonika ausprägt. Die in Takt 13/14 herausgegriffenen Töne $b$ und a (Quintton und Grundton der Tonika) geben hier die Richtung an. Im Original steht die erste Eintragung »D« (Dominante) fälschlich über $g^{2}$, die zweite über $\operatorname{cis}^{1}$ (T. 13).

68 Typoskript: in T. 21 (sic).

69 T. 18 - Anfang 22.

70 In Notenbeispiel 40 sind diese letzten beiden Töne der Tonika, $g$ und dis, durch die Ziffern 7 und 8 bezeichnet.

71 a-d-a-gis (T. 27/29) ist Subdominante, a-e-a-g (T. 28, 29, 2. Hälfte) Tonika; c-es-c-b (T. 4/5) ist ebenfalls Tonika.

72 Vgl. Notenbeispiele 33 und 34.

73 Nämlich Bindeglied zwischen Kleinterzgruppen. - Es folgen recht allgemeine Reflexionen über verschiedene Arten, mittels verschieden großer Kleinterzgruppen (aus 2, 3, 4 Tönen), die zueinander in verschiedenen Intervallen stehen, den Zwölftonraum zu erfüllen. 
Vollständige Kleinterzgruppen mit großen Terzen verbunden. ${ }^{74}[\ldots]$

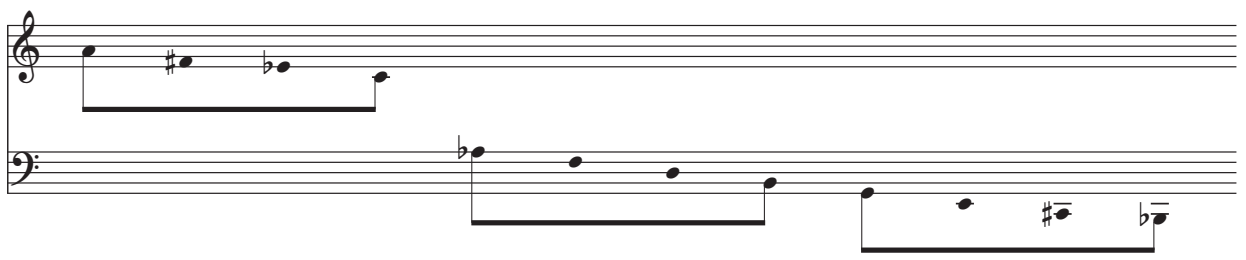

Beispiel 41

Man kann auch je zwei Töne in Kleinterzabstand durch große Terzen verbinden. ${ }^{75}$

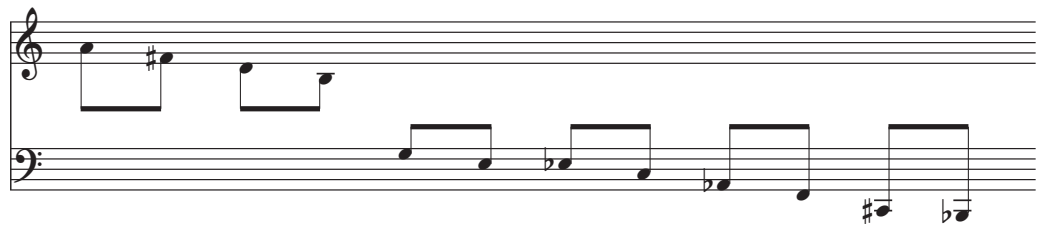

Beispiel 42

NB. Kleine Sekunde als Bindeglied. ${ }^{76}$

Die Kleinterzgruppen können unterschiedliche Anzahlen von Tönen enthalten. ${ }^{77}$

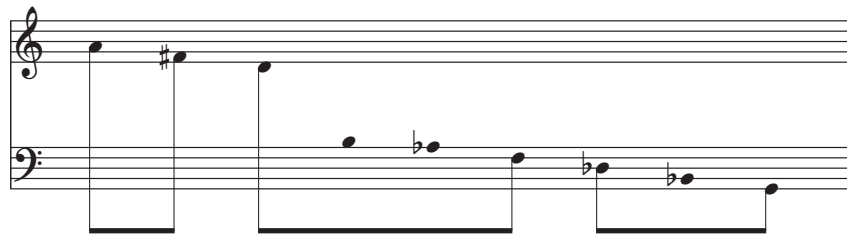

Beispiel 43

Kombinationen verschiedener Kleinterzverbindungen. Die Kontrolle hat die Quinte. ${ }^{78}$

74 Vollständige Kleinterzreihe = vier Töne in Kleinterzabstand, alle Grundtöne einer Funktion.

75 Notenbeispiel 42 enthält zwei Hexatone: $g$ bis fis und des (cis) bis c, zusammen also alle zwölf Töne.

76 Die kleine Sekunde e-es ist Bindeglied zwischen den beiden Hexatonen.

77 Beispiel 43 enthält die Töne der Takte 30/31.

78 Beispiel 44 bezieht sich offenbar auf Takt 30/31. Die erste Fünftongruppe, ein Hexaton $g$ bis fis ohne e, gibt ebenso eine Tendenz der Takte 30/31 an (T. 30 erste Hälfte plus der erste Ton von T. 31), wie die zweite Tongruppe, Konstrukt IIb (die ersten drei plus die letzten drei Töne von T. 30), vgl. auch Anmerkung 63. In sämtlichen Tonreihen von Beispiel 44 werden die kleinen Terzen von indirekten Quinten festgehalten. 


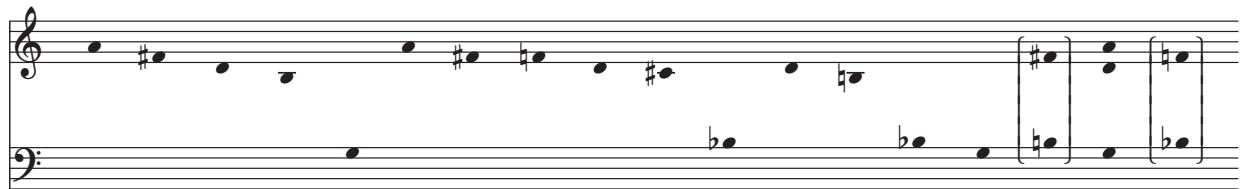

Beispiel 44

Die folgenden sechs Takte ${ }^{79}$ leiten einen Fakturwechsel ein in der Behandlung der Kleinterzeinheiten. Die großen Terzen treten auf und übernehmen die Funktion der Quarte. ${ }^{80}$ Auch die Anzahl der Töne in den Kleinterzgruppen wird unterschiedlich.

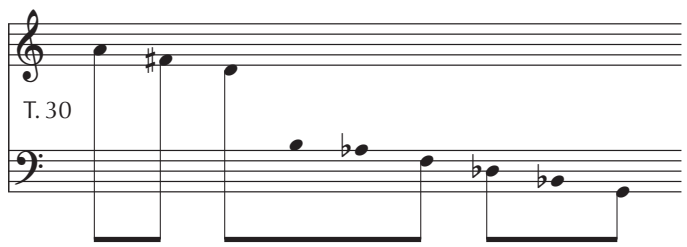

Beispiel 45

[...] Mit einer Spiegelung ${ }^{81}$ kommen neue Töne hinzu, die nichts anderes sind, als die weiteren Töne der jeweiligen Modi: oben (r. H.) Subdominant-, unten (I. H.) Dominantgruppe.

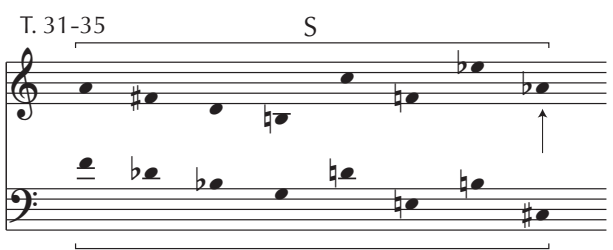

$\mathrm{D}$

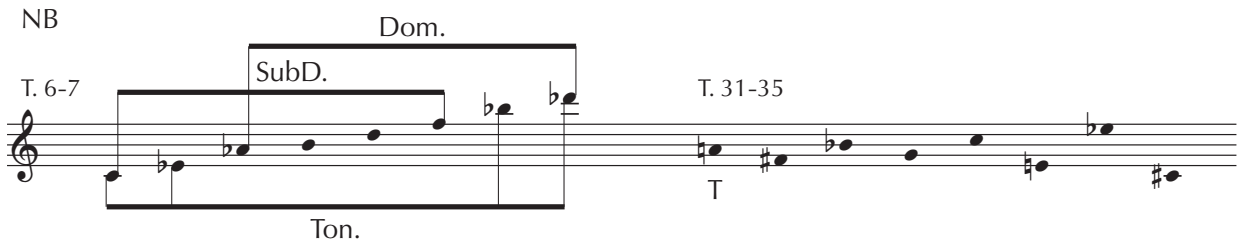

Beispiel 46

79 T. 30-35.

80 Es werden hier die Takte 30-35 mit den Takten 6-13 verglichen. In Takt 6-13 werden Gruppen aus zumeist vier Tönen in Kleinterzabstand durch Quarten verbunden (z. B. $f-b$ T. 6/7; $f$ kommt von as$h-d ; b$ geht weiter mit des). In Takt 30-35 werden verschieden große Kleinterzgruppen durch große Terzen verbunden (z. B. fis-d, f-des T. 32; fis kommt von a, $d$ geht nach $h$; des kommt von $g$ - $b$ ). In dieser Hinsicht übernehmen die großen Terzen ab Takt 30 die Funktion der Quarten ab Takt 6.

81 Mit 'Spiegelung` ist hier die Gegenbewegung der beiden Hände bei der Erweiterung ihres Tonvorrats gemeint (T. 32-36 Anfang). 
NB. Die untere Gruppe (D) erhält zur Hilfe aus der oberen Gruppe (S) ein as als achten Ton.

NB. Die Rahmentöne der Arpeggien ergeben die Tonikagruppe. ${ }^{82}$ Dies ist ein Nebenprodukt, das aus der Subdominant- und Dominant-Bimodalität zustande kommt. Das erste Arpeggio Takt 6-7 enthält auch im Wesen diese bewegliche Gemeinschaftlichkeit der Tongruppen. ${ }^{83}$

Die Komplexität solcher Art von Spiegelbewegungen der unabhängigen Modi führt zur Darstellung der funktional bestimmten Tongruppen insgesamt ${ }^{84}$ : natürlich ist die dritte Gruppe auch da, wie eine mögliche dritte Perspektive aus den beiden, also ist die Stelle in diesem Sinne (wenn man will) polymodal.

Takt 36-38: Was jetzt folgt, ist ein Gang von mehrfacher Bedeutung. Polysemantisch, würde man am besten sagen. Doch er rückt - eindeutig - das Ganze zur Tonika.

Die Bassgrundtöne sind eine lineare Tonika. ${ }^{85}$ Plötzlich springen diatonische Segmente ein, die einander folgen bis $h-e-a .{ }^{86} \mathrm{Im}$ Nacheinander ist e der letzte, der mit einer Elision folgt als Schluss, eine Rekapitulation der ersten Folge c-e-gis ${ }^{87}$; jetzt e-gis-c.

Die Änderungen der oberen Stimme - weil die untere ihre Struktur behält ${ }^{88}-$ dient zweimal harmonischen Zwecken, $\mathrm{D}^{89}$ steht für die Dominante, $\mathrm{DES}^{90}$ für die Tonika, und beim letzten Mal äußert sich eine eigene Logik der zwei großen Terzen: $h$, e-gis-c. ${ }^{91}$ Und ihre Ausgangstöne sind die diatonischen Segmente $c h g d / f^{92}, h$ a e gis $c .^{93}$

NB. Außerdem sind die ersten Töne ein bestimmter Gang zu b. Also cis, $c, h$, $b$-g-es- $c^{94}$ eine Rückleitung zur sthematischen Tonikagruppe.

82 Beispiel 46, drittes System.

83 NB unter Beispiel 46: die ersten sechs Töne $c$, es, as, $h, d, f$ gehören zur Subdominante, die letzten sechs Töne as, $h, d, f, b$, des zur Dominante und die zwei ersten mit den zwei letzten Tönen $c$, es, $b$, des zur Tonika (die Dominantlinie im Notenbeispiel beginnt im Original zu weit links, bei $c^{1}$; sie müsste bei $a s^{1}$ beginnen). Man könnte auch die ersten drei plus die letzten drei Tönen $c$, es, as, $f, b$, des als Hexaton des bis $c$ deuten, die Töne dazwischen, $a s, h, d$, $f$, als die vier Subdominantgrundtöne. Vgl. dazu die Tonreihe Takt 30 mit Konstrukt Ilb a-fis-d, $f$-des-b und dazwischen liegend die vier Grundtöne der Subdominante $d$ - $h$-as-f.

84 Aller drei Funktionen.

85 (cis-) dis-e-fis (T. 36/37).

86 T. $37 / 38$, in der Reihenfolge $h$-a-e.

87 Gemeint ist wohl c (T. 6) - e (T. 8) - gis (T. 10).

88 Die Struktur der unteren Stimme ist die eines 'halbverminderten Septakkordes` (vgl. T. 32, 33, 34, 35, 36 erste drei Achtel, 37 erste Hälfte mit Auftakt etc.); dagegen behält die obere Stimme nicht die Struktur eines Mollseptakkordes bei (vgl. T. 33 erste Hälfte + Auftakt, T. 34 erste Hälfte + Auftakt etc. mit T. 36 letzte drei Achtel, T. 37 erste drei Achtel etc.).

89 Der letzte Ton der r.H. in Takt 36, im Zusammenhang ein Quintton der Dominante (in genauer Sequenz zum Bisherigen müsste dis stehen).

90 Der vorletzte Ton der r. H. in Takt 37, im Zusammenhang ein Quintton der Tonika.

91 e-gis-c wiederholt fis-ais-d (T. 36) und f-a-des (T. 37).

92 T. 37, ein Pentaton.

93 T. 38 mit Auftakt, fünf Töne eines harmonischen Heptatons.

94 cis (T. 36), $c$ und $h$ (T. 37), b (T. 39); die Tonikagruppe b-g-es-c (T. 39-43). 


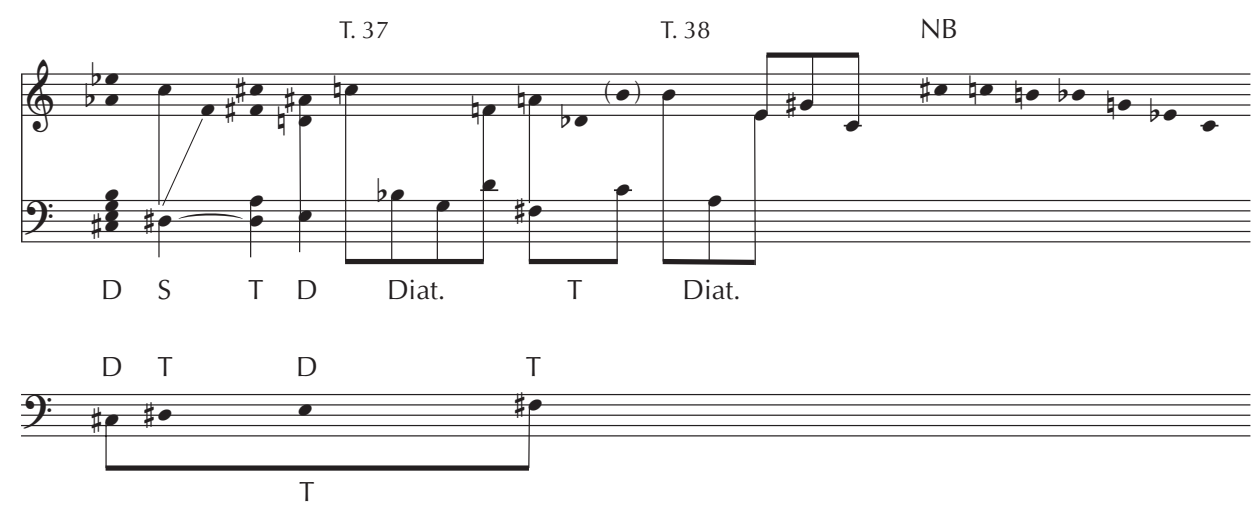

Beispiel 47

Eine bimodale Situation. ${ }^{95}$ Eine Reprise von Takt 30, aber jetzt als Tonika und Subdominante.

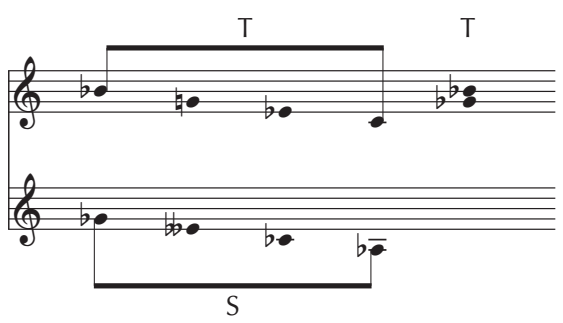

Beispiel 48

Die Rahmentöne der beiden Hände im Zusammenklang (T. 44).

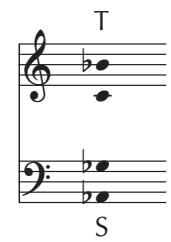

Beispiel 49
Es rückt zur Tonikagruppe.
Ein Wechsel zur

Dominante (T. 45).

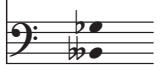

Beispiel 50

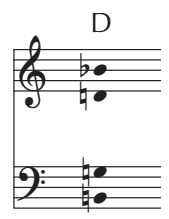

Beispiel 51

95 T. $39 \mathrm{ff}$. 
Plötzlich ein sehr kurzer Tonika-Akkord. Es sind vier Noten aus den fünf Anfangstakten (vgl. die vier Rahmentöne aus Beispiel 36, Spalte 1).

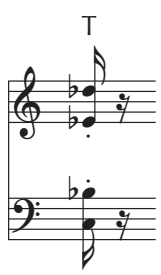

Beispiel 52
Und ein starkes Tonikasegment (T. 46).

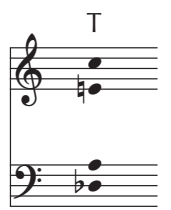

Beispiel 53
Takt 47: Es folgt eine Umformung des Akkordes.

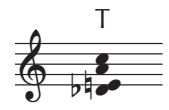
Dieser Tonikaklang ist der Beginn des ersten Arpeggios der Reprise.

Beispiel 54

Zur pentatonischen Melodie entfaltete Rekapitulation der formalen Funktion von Takt 4-5 (T. 48-49).

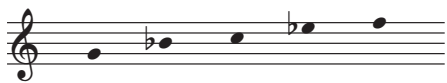

NB

$\begin{array}{ll}\text { T. } 48 & \text { T. } 77\end{array}$

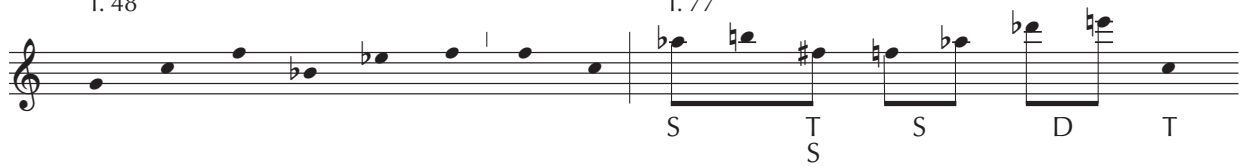

Beispiel 55

NB. Ein Pentaton, also ein Segment der diatonischen Reihe, ist äußerst beweglich. Der letzte Ton deutet die Richtung der Subdominante an, die sich plagal sofort zurückwendet zur Tonika. ${ }^{96}$ Dieser plagale Effekt wird betont und breit ausgesagt im Schlussteil, und aufgrund dessen folgt das [...] aus dem Grundmaterial aufgebaute, authentische Aussprechen von $c$ als Grundton. ${ }^{97}$ Dieser [...] Prozess hat gleichzeitig einen fühlbaren [...] tonikalen Inhalt. Er gehört zur breiten, umfassenden Tonikalität, zur Erwähnung von fis-C (T. 46-47) und früher von dis-a (T. 25-26).«-

»Takt 50: Die Reprise bringt das Thema in Umkehrung, auch die Richtung der Sequenz ist umgekehrt, aber die Geschlossenheit der 24 Töne bleibt.

96 Das $f$ (Subdominante) am Ende von Takt 49 wendet sich zur Tonika am Anfang von Takt 50.

97 NB. zu Beispiel 55 zeigt zuerst den Tonvorrat Takt 48/49 quintenweise aufgeschlüsselt: $g$-c- $f-b$-es. Ab Takt 77 findet ein (plagaler) Weg von der Subdominante (as- $h$-fis, $f$-as) zur Tonika $c$ statt. Dadurch, dass das dominantische Segment des-e zwischen Subdominante und Tonika gestellt wird, ist die Abfolge dort letztlich doch authentisch. 


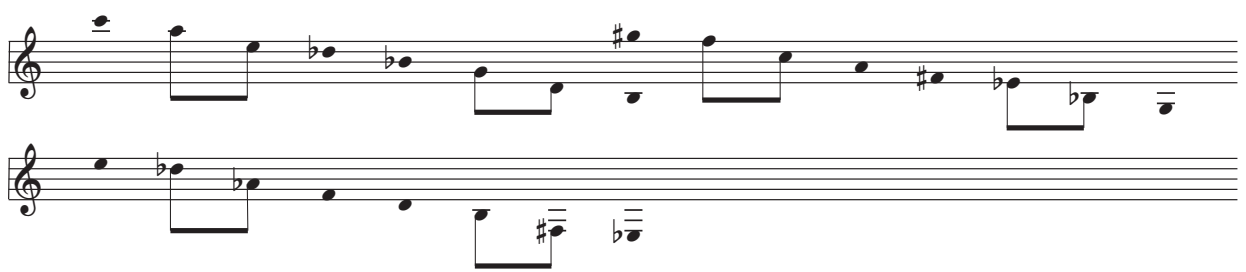

Beispiel 56

Die Überleitung zur Einschränkung auf die Subdominante ist abgekürzt, aber graduell regelmäßig laufend ins neue Arpeggio eingeschmolzen. ${ }^{98}$

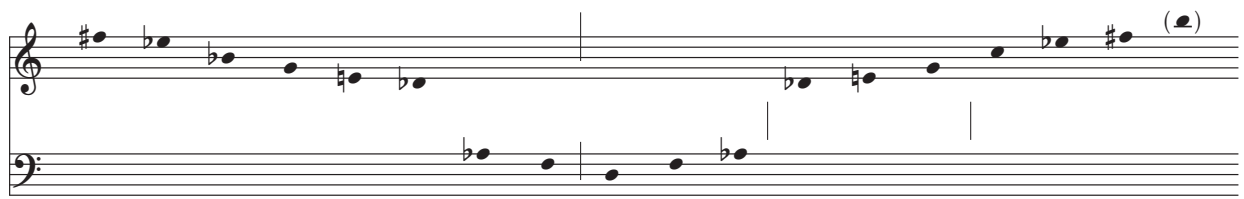

Beispiel 57

Dieselbe Prozedur ${ }^{99}$ wie im Miniatur-Schlusssatz der Tonika-Exposition (T. 22). Hier klare Subdominante.

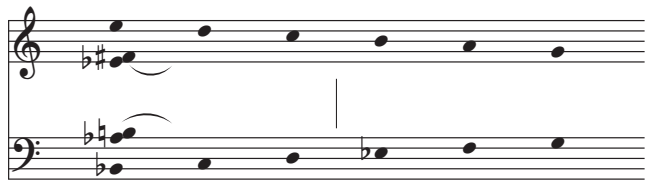

Beispiel 58

Wiederholter S-Klang ${ }^{100}$ und hinzugefügt der erste S-Teil des ersten Arpeggios als Bestätigung. Die plagale Wendung nach fis ist vorläufig als Subdominante zu verstehen. ${ }^{101}$

98 T. 55-57. Ab Takt 56, zweites Sechzehntel, besteht das Arpeggio aus $3+3+3$ Tönen (siehe die kleinen Striche in Beispiel 57): $d$-f-as (Subdominante), des-e-g (Dominante), c-dis-fis (Tonika bzw. Quinttöne der Subdominante). Es handelt sich also um dreitönige Kleinterzgruppen in Quartabstand.

99 In den Takten 63-66.

100T. 67.

101 Gemeint ist wohl der Schritt $h$-fis Takt 69 mit Auftakt sowie Takt 71 Mitte, vgl. dazu den Schritt $f$-c T. 50 mit Auftakt. fis T. 69 bzw. 71 ist trotz dieser plagalen Wirkung als Subdominante (Quintton), nicht als Tonika zu verstehen, vermutlich weil die Takte 66-74 ausschließlich Subdominanttöne enthalten (zu einer vollständigen Subdominante fehlen $f$ und a). Da wäre es wenig einleuchtend, einzig diesem fis den Subdominantstatus abzusprechen. 


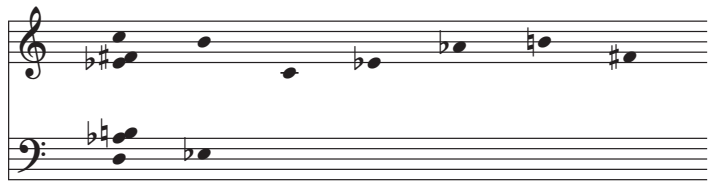

Beispiel 59

Ganz geklärter S-Klang. ${ }^{102}$

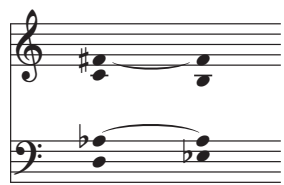

Beispiel 60

Plötzlich im Piano das ganze sexponierende` Arpeggio wiederholt. ${ }^{103}$

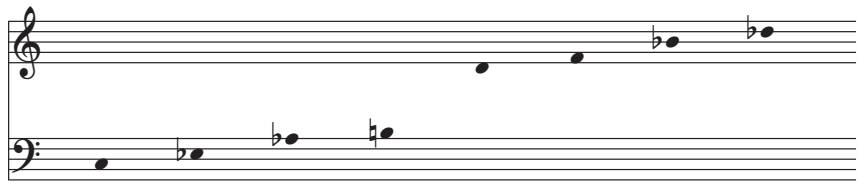

Beispiel $61^{104}$

Einmal die Subdominant-Einheit mit fis. Unmittelbar hinzugefügt (crescendo - rallentando) eine Dominant-Einheit, die aus S + D besteht ${ }^{105}$ und die unmittelbar zum Grundton führt: $c$. Diesem Grundton ist eine Tonika-Einheit hinzugefügt, bestehend aus D + T. ${ }^{106}$

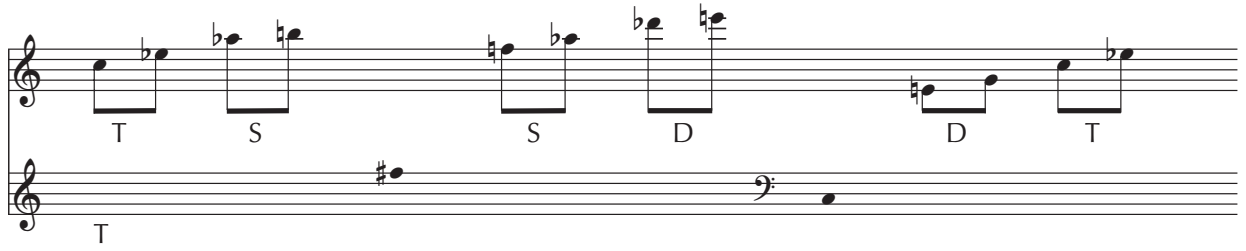

Beispiel $62^{107}$

102 T. $72-74$.

103 T. $74 / 75$.

104 Im Original steht Notenbeispiel 61 irrtümlich unter dem nächsten Absatz.

105 D.h. die aus Quint- und Grundtönen der Dominante besteht.

106 D. h. aus Quint- und Grundtönen der Tonika. 
Die kleinsten Elemente bis zum Einzelton abgebaut ${ }^{108}$ können nur den wirklichen Sinn eines Systems rechtfertigen. - Zur Wahrheit muss der Weg auch wahr sein.»

In seiner Analyse von Staccato (Mikrokosmos Nr. 124) weist Albert Simon in seinem Beispiel 15 unter NB auf zwei Quartenreihen hin, aus denen das Grundmotiv $d^{1}$-cis ${ }^{1}$-a und seine Transpositionen (teils in Umkehrung) gebildet sind. Es handelt sich um ein Pentaton $g$ bis $h$ und ein weiteres des bis $f$. Diese sind um zwei zentrale Tritone ( $d$ bis e und as bis $b)$ gruppiert, die den vier Erscheinungsformen des Grundmotivs entsprechen: $d$-a (Grundmotiv), e-a (Umkehrung T. 17), Ais-dis (Umkehrung T. 11/12), gis-es (Grundmotiv T. 12). Aus den beiden Pentatonen kann man sämtliche Töne dieser vier Themengestalten inklusive ihrer Oktavlage ableiten (vgl. Beispiele 1-4). Dabei zeigt sich, dass man das Pentaton des bis $f$ als reine Quartenreihe von $F$ bis $d e s^{1}$ vorstellen muss. Das Pentaton $g$ bis $h$ reagiert auf das erstgenannte, indem dort auf Halbtonanschlüsse im Vergleich zu jenem geachtet ist: $F \rightarrow E, B \rightarrow H$, as $\rightarrow g$, des $\rightarrow d$, der mittlere Ton es wird zum mittleren Ton a des zweiten Pentatons. Damit sind sämtliche Töne der vier Gestalten des Grundmotivs abgeleitet (siehe Beispiel 63). Die durchgezogenen bzw. die gestrichelten Linien in Beispiel 63 deuten die Herkunft des Grundmotivs und seiner Umkehrung aus den in Tritonen bestehenden Pentatonen an.

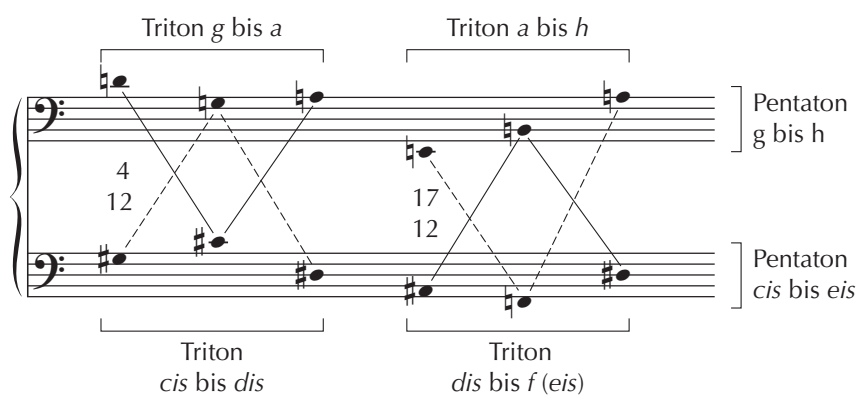

Beispiel 63

Simon weist darauf hin, dass in den Takten 23-26 ein freier Krebs des Grundmotivs auftaucht (Beispiele 18 NB und 25c). Dort wird zugleich der letzte im Stück fehlende Tritonus, c-fis erreicht (vgl. Beispiel 14 NB mit Kommentar, vgl. auch Beispiel 15 NB mit den in Klammern hinzugefügten Tönen $c$ und fis). Analog zu Beispiel 63 kann man die beiden Krebsgestalten des Grundmotivs darstellen wie in Beispiel 64 (vgl. Beispiel 18 NB).

107 Dieses Beispiel fehlt im Original. Es lässt sich aber aus dem Text mühelos rekonstruieren (vgl. auch Beispiel 55 unter NB, ganz rechts).

108 »abgebaut« = analysiert. 


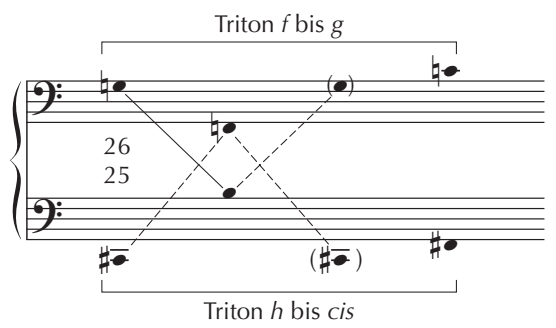

Beispiel 64

Sie sind aus zwei Tritonen gebildet, die einerseits die eben genannten Pentatone zur Zwölftönigkeit ergänzen und andererseits die vier Tritone, aus denen zusammengesetzt man jene Pentatone auffassen kann, zur Vollständigkeit bringen. Jedes Triton hat mit seinem Nachbartriton einen Ton gemeinsam, folglich braucht man sechs Tritone, um alle zwölf Töne abzudecken. Diese Konstruktion, zwei Pentatone ( $g$ bis $h$ und des bis $f$, bestehend aus je zwei Tritonen) plus zwei Tritone ( $f$ bis $g$ und $h$ bis cis) sind ein Zwölftonfeld und das erste Tonfeld des ganzen Stücks. D. h. das erste Tonfeld des Stücks ist die Summe der Beispiele 63 und 64.

Hinsichtlich des Grundmotivs bestehen zwei Inkonsistenzen. 1. Das Grundmotiv taucht sehr deutlich in Originalgestalt und in Umkehrung auf, etwas verdeckt im Krebs. Müsste es nicht auch in Krebsumkehrung auftauchen? - 2. Wenn man die Halbtonschritte des Grundmotivs gemäß ihrer funktionalen Zugehörigkeit analysiert, ergibt sich Folgendes: Alle Halbtonschritte des Grundmotivs in Originalgestalt und in Umkehrung ergeben zusammen eine vollständige Dominante: $d$-cis, Ais-H (T. 11/12), gis-g (T. 12), E-F (T. 17). Die Halbtonschritte des Motivs im Krebs entstammen alle der Subdominante: F-Ges (T. 23/24) $)^{109}$ sowie $h-c^{1}$ (T. 26). Es fehlt aber ein Halbtonschritt im Grundmotiv, der die Tonika ausprägen würde.

Beide Inkonsistenzen verschwinden zugleich. Die sReprise ab Takt 27 Mitte ist sehr ausgeprägt. Ab dort dominiert die Originalgestalt des Grundmotivs $d$-cis-a, die jeweils von der Umkehrung ais- $h$-dis kontrapunktiert wird. Immer beginnt aber die Originalgestalt (T. 27 Mitte, T. 31 mit Auftakt, T. 33 mit Auftakt) und erweist sich so als führend. Daran schließt sich eine Passage an, in der die Intervalle des Grundmotivs sukzessive verkleinert werden, bis am Ende - sehr insistierend - nur der Halbtonschritt $b$-a übrigbleibt (ab T. 35). Die charakteristischsten Ereignisse dort sind demnach das $d$ (ab T. 27) und weiterhin jener Halbtonschritt $b$-a. ${ }^{110}$ Diese Tonfolge $d$ - $b$-a ist nun zugleich die gesuchte Krebsumkehrung des Grundmotivs, und sie enthält den ebenfalls gesuchten Tonika-Halbtonschritt. (Albert Simon weist darauf hin, dass sich in der Schlusspassage ab Takt 33 besonders viele tonikale Klänge befinden: Beispiel 12 und Kommentar dazu, vgl. auch Beispiele 9 und 10.)

109 Es ist hier deutlich, dass es keinen Unterschied gibt zwischen $F$ und Eis, zwischen Ges und Fis, vgl. T. 24 und 25, linke Hand.

110 Unter bzw. über dem a in den Takten 28, 31, 33 steht jeweils ein ais, das eine (plagale) Tendenz zu einem $h$ hat - tatsächlich folgt auf jedes ais hier ein $h$. Diese Tendenz wird erst mit den $b$ ab Takt 35 (bzw. schon T. 34) umgekehrt: Diese streben (authentisch) je zu einem a und bereiten auf diese Weise den Schluss vor. 
Der Schluss wird von dem Intervall $b$-a bestimmt; kaum weniger charakteristisch ist aber der Kontrapunkt gis-a, der die simultane verminderte Terz gis/b hervorbringt (der vorletzte Klang des Stücks), die hier tatsächlich als solche gehört werden kann (vgl. Beispiele 12 und 13, je Ende). Ein ähnliches Intervall gibt es am Anfang des Stückes: Das erste simultane Intervall in Takt 4 ist es/cis, eine übermäßige Sexte (vgl. Beispiel 1). Bei näherer Betrachtung bemerkt man, dass die Tonvorräte am Anfang und am Ende ähnlich, aufeinander bezogen sind. Den Anfang machen es, $d$, cis (als dritter Ton kommt ein a dazu), am Ende stehen gis, $a, b$; als weiteren besonders relevanten Ton kann man das oben erwähnte $d$ hinzunehmen (dann antwortet das Motiv $d$-b-a dem Motiv $d$-cis-a). Diesen Tonvorrat kann man zusammengenommen darstellen wie Beispiel 65a zeigt.

a)

b)

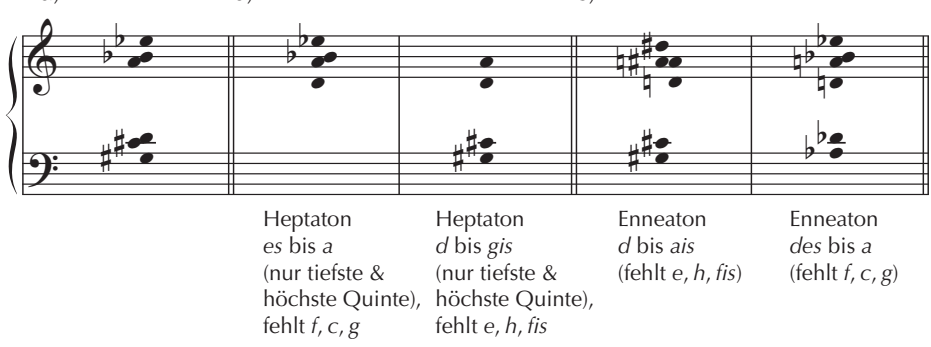

Beispiel 65

Diese sechs Töne kann man wiederum zerteilen in zwei Heptatone, die jeweils nur durch ihre tiefste und ihre höchste Quinte repräsentiert werden (Beispiel 65b). Auf diese Weise wird der Bezug von es und cis, b und gis gekappt. Vielleicht ist es daher notwendig, auch die Darstellungen von Beispiel 65c zu berücksichtigen. Hier sind die sechs Töne als unvollständiges Enneaton gedeutet, in zwei einander widersprechenden Weisen, die aber präzise gleich stark sind, weswegen es unmöglich ist, eine Version vorzuziehen. Ihr Widerspruch gegeneinander und ihr Bezug aufeinander ist wohl das Entscheidende hier. Entweder handelt es sich um ein Enneaton von $d$ bis ais, bestehend aus den Tönen 1, 2 sowie 6 bis 9 ( $e$, $h$, fis fehlen also) oder um ein Enneaton von des bis a, bestehend aus den Tönen 1 bis 4 und 8, 9 ( $f, c, g$ fehlen also). Dieses gedoppelte Enneaton, das doch nur eines ist, ist das zweite Tonfeld des Ganzen der vorliegenden Komposition. ${ }^{111}$

Ein untergeordneter Aspekt unterstreicht den Bezug des Schlusses auf den Anfang: Der erste Halbtonschritt $d$-cis des Stücks eröffnet als plagaler Schritt: Plagal ist er wegen des darunterliegenden es: es/d ist Subdominante, es/cis Tonika. Der letzte Halbtonschritt des Stücks schließt als kraftvoller authentischer Schritt: gis/b ist Dominante, a/a Tonika.

Anhand der Passage Takt 12-19 soll kurz gezeigt werden, wie die Intervalldisposition von Beispiel 63 auch auf andere Aspekte als die Disposition des Grundmotivs wirkt. Beispiel 66 zeigt die Passage im Groben aufgeschlüsselt.

111 Damit wird zugleich der besondere Charakter der verminderten Terz Takt 36 Ende bzw. der übermäßigen Sexte Takt 4 angegeben: Sie vagieren zwischen verminderter Terz und großer Sekunde etc. 


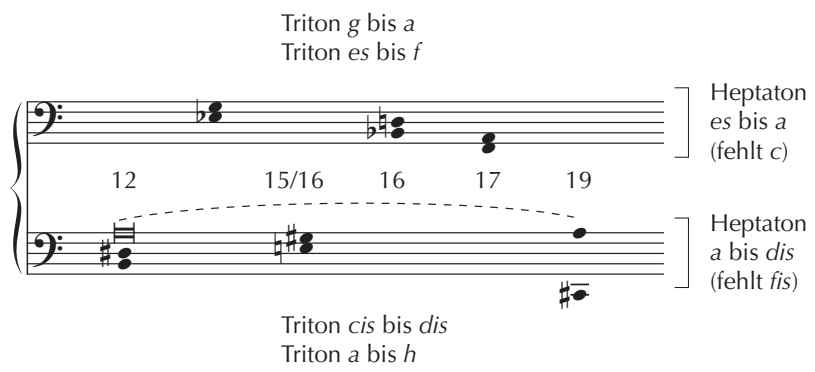

Beispiel 66

Hier finden sich die bereits aus Beispiel 63 bekannten Tritone zu Heptatonen verbunden wieder. Auf die Terz g-es (T. 12) antwortet die Terz $d$-Ais (T. 16, vgl. Simon, Beispiel 24) und weiterhin die Terz F-A (T. 17). Ähnlich reagiert die Terz as-fes (enharmonisch verwechselt geschrieben, T. 15/16, siehe Simon, Beispiel 24) auf die Terz H-dis (T. 12) und wird ergänzt durch das Cis in Takt 19, zu dem sich das orgelpunktartige a ab Takt 12 gesellt. Damit ist die Entwicklung dieser Passage im Groben beschrieben. Albert Simons Beobachtung der Dominantreihe in Takt 16 mit Auftakt (Beispiel 7) findet ihre Begründung im Zusammentreffen der Terzen gis-e und $d$ - $B$ (enharmonisch anders geschrieben), die beide Quintenreihen entstammen, an dieser Stelle aber zusammen eine Dominante ausprägen - diese Koinzidenz wird von der Dominantreihe ausgebaut (ich spreche in solchen Fällen von einer seffizierten Dominante: Die Dominante ist nicht ursprünglich dort, sondern sie wird von etwas ganz anderem wie beiläufig veranlasst).

Bei den nicht thematischen Ganztonstellen (siehe Beispiel 14 NB und Simons Kommentar dazu) finden sich ausschließlich Gruppen aus den Tönen $g, a, h$ sowie des, es, $f$ (cis, dis, eis). Das sind die einzigen Ganztongruppen, die innerhalb von je einem der in Beispiel 63 genannten Pentatone existieren; bei anderen Großterzgängen müsste man die Pentatone vermischen. Dies ist ein weiterer Hinweis auf die Relevanz der beiden Pentatone für das Stück.

Die einzige Quarte des Pentatons $g$ bis $h$ (Beispiel 63), $a-d^{1}$, spielt offensichtlich im Stück eine besondere Rolle, vgl. Simons Beispiele 5, 15 (3. System), 22, 23. Die dort beobachtete Bedeutung dieser Quarte und ihre Interpretation als Auslöser eines subdominantischen Tetrachords $(a-h-c-d)$ und anderer Ausfüllungen kann man als vom ersten Tonfeld des Ganzen abhängig verstehen.

In einer kürzlich erschienenen Studie von Edward Gollin werden Beobachtungen zu Geteilte Arpeggien (Mikrokosmos Nr. 143) mitgeteilt ${ }^{112}$, die eine gewisse Nähe zu Teilen aus Albert Simons Analyse haben. Gollin interessiert sich - im Sinne der transformational theory - dafür, wie oft man einen Zyklus von Intervallen (z. B. kleine Terz - Quarte 
- kleine Terz - kleine Terz, vgl. T. 6 ff.) wiederholen kann, bis man die pitch-classes seines ersten Auftretens wiedererhält. Die Takte 6-13, 50-57 und auch 30-32 sowie 39-43 liefern hier reiches Anschauungsmaterial. Man erkennt schnell, wie viel von Gollins Ideen zur Intervallsystematik in Simons mindestens zwanzig Jahre älterer (unveröffentlichter) Analyse vorweggenommen ist. Albert Simons Beispiele 31 bis 33 sowie seine Bemerkungen zu Beispiel 37 und vor allem zu den Beispielen 41 bis 44 (beginnend mit der Bemerkung zu Takt 30) betreffen die möglichen Reihen aus kleinen Terzen mit je anderen Intermediärintervallen. Exakt dasselbe stellt Gollin in seinen Examples 17 und 20 dar. ${ }^{113}$ Gollin weist im Unterschied zu Simon (Beispiele 34, 35) nicht ausdrücklich auf den Zusammenhang der Quarten in der Passage Takt 6-11 hin und auch nicht auf die daraus resultierenden beiden vollständigen Ganztonreihen (Simon Beispiel 35 NB), da die Frage nach dem Bezug nicht nebeneinander stehender Töne nicht zur Fragestellung von Gollins Artikel gehört. An die Grenze seiner Methode gelangt Gollin in seiner Erläuterung eines Tones aus Takt 56. ${ }^{114}$

Auf Albert Simons Analyse aufbauend kann man die Passagen Takt 6-26 und Takt 46 (50)-67 folgendermaßen in Beziehung setzen. Die Takte 14-26 komponieren den Klang cis/e/fis/a aus, die Takte 57-67 den Klang dis/fis/gis/h, d. h. diese Töne verfestigen sich in den genannten Takten (andere treten hinzu). Ähnlich kann man in den Takten 6-13 die Töne $c$, es, as, $h$, in den Takten 46-56 die Töne des, e, a, c als die führenden behaupten. Mit $c$, es, as, $h$ (T. 6) ist das Prinzip dieser Passage gegeben: Kleine Terzen werden von Quarten zusammengebunden. Die von Simon genannten Ganztonreihen (Beispiel 35 NB) lassen sich als zwölftönige Verbindung von der Quarte es/as des Klangs c/es/as/h zur Quarte cis/fis des Klangs cis/(e)/fis/a (T. 10/11 bzw. 14) deuten. Demnach ist cis/e/ fis/a in den Takten 10/11 eine Vorandeutung des Klangs ab Takt 14 (vgl. I. H. Takt 14 mit r. H. auf der Wende von Takt 10 nach 11). In der Reprise ab Takt 50 ist alles spiegelsymmetrisch analog (vgl. Beispiel 56). Aus diesen Überlegungen ergibt sich Beispiel 67.

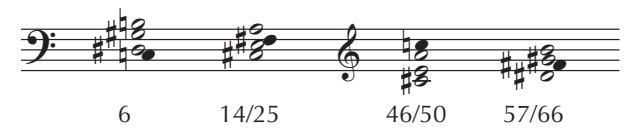

Beispiel 67

113 Simons Notenbeispiel 37 kommt mit Gollins Example 17 (160) überein, Simons Notenbeispiel 43 mit Gollins Example 20 (162), wobei Simon Noten schreibt, Gollin Tonnamen (pitch-classes). Simon diskutiert in seinen Notenbeispielen 42 und 44 überdies noch andere Kombinationsmöglichkeiten von kleinen Terzen und anderen Intervallen, die durch ihr Verhältnis zur Zwölftönigkeit bestimmt werden.

114 Nach Gollin müsste der letzte Ton von Takt 56 ein $b^{1}$ statt eines $c^{2}$ sein (vgl. sein Example 17 auf Seite 160, „Odd series«). Eine regelmäßige Intervallfolge (Gollin betrachtet nur ausgewählte regelmäßige Intervallfolgen), die mit $c$-a-e-cis- $b$ beginnt, müsste später die Töne fis-es- $b$-g-e enthalten. Auf Seite 162 nennt Gollin den Ton $c^{2}$ in Takt 62 »an sout-of-place « note « und versucht ihn zu erklären, indem er sich von seiner Theorie regelmäßiger Tonreihen unabhängig macht. Vgl. dagegen Simons Notenbeispiel 57 und seinen Kommentar dazu: Simon ist nicht genötigt, zur Erklärung dieses Tons eine Irregularität zu behaupten. 
Die beiden Akkordpaare sind genau spiegelsymmetrisch, Spiegelachse ist $c^{1}$, der Grundton des Stücks. Es handelt sich um ein Heptaton a bis dis, das um fis zentriert ist (also kein E-Dur). Dem Zentrum fis ist ein c hinzugefügt, so dass das Tonfeld acht Töne aufweist. ${ }^{115}$ Beispiel 68 zeigt denselben Tonvorrat als Tonleiter.

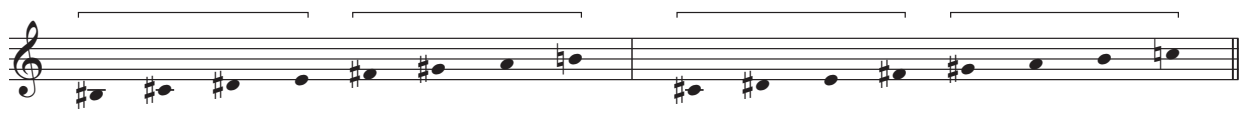

Beispiel 68

Die Spiegelbildlichkeit der Takte 50-67 im Verhältnis zu Takt 6-26 und zugleich ihr enger klanglicher Bezug aufeinander ist in Beispiel 67 begründet.

Der Mittelteil (T. 30-45) führt ein Oktoton aus, dessen mittlere Quarte c- $f$ fehlt. Der Bezug von as/es $/ b^{1}$ (T. 39) auf $g / d^{1} / a^{1}$ (T. 30 ff., Beispiel 44 rechts außen) ist im Original leicht zu hören. Der erste Klang des Oktotons wird folgendermaßen symmetrisch ausgeführt:

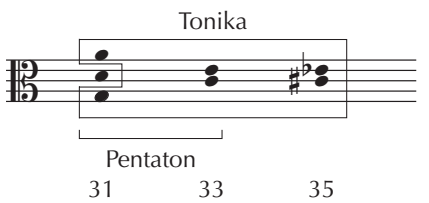

Beispiel 69 und näherhin so:

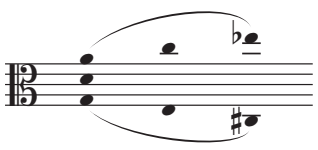

Beispiel 70

Diese Tonika entspricht Simons Beispiel 46, 3. System. ${ }^{116}$ Der zweite Klang as/es $1 / b^{1}$ (T. 39) scheint in seiner Fortführung zu einem Hexaton erweitert werden zu sollen. Auf

115 Man kann sich ein symmetrisches sechstöniges Heptaton (gis-Moll plus A-Dur) vorstellen (dies ist die einzige Möglichkeit, dieses Heptaton sechstönig und symmetrisch darzustellen), dessen einzigem Tritonus die weiteren Töne derselben Funktion zugefügt sind.

Das hinzugefügte $c$, der Grundton der Komposition, spielt eine Sonderrolle. $c^{0}$ ist ab Takt 6 die untere, $c^{3}$ ab Takt 50 die obere Grenze des Tonraums.

Es liegt eine besondere schöne Subtilität in der Tatsache, dass das gis-Moll am Ende der Reprise (letzter Akkord Beispiel 67) dem A-Dur des Anfangs (zweiter Akkord) und das A-Dur der Reprise (dritter Akkord Beispiel 67) dem gis-Moll des Anfangs (erster Akkord) symmetrisch entspricht.

(Das genannte Tonfeld kann auch interpretiert werden als ein Pentaton e bis gis, dessen mittlerer Ton fis zum Grundton erklärt wird und von seinen sämtlichen kleinterzäquivalenten Grundtönen ergänzt wird. So kommen c, a, dis hinzu. Die letztgenannten beiden Töne ergänzen das Pentaton e bis gis zum Heptaton a bis dis.)

116 Nach meiner Meinung stehen die Töne fis ${ }^{1}$ und $b^{1}$ in Simons Notenbeispiel 46 nicht auf derselben Ebene wie die übrigen. 


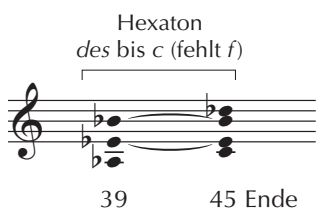

Beispiel 71

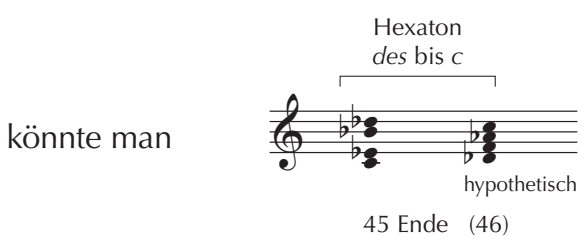

Beispiel 72

erwarten, was das Hexaton vervollständigen würde. Die tatsächliche Fortführung

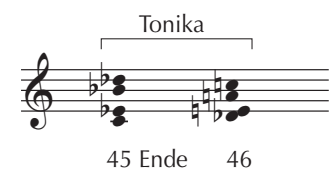

Beispiel 73

erbringt den dritten Akkord von Beispiel 67 (dem ersten Tonfeld des Ganzen). Zugleich erweist sich der letzte Akkord Takt 45 hiermit als die Schnittmenge des Hexatons des bis c mit der Tonika. Zu Recht also insistiert Albert Simon auf der Bedeutung dieses Akkords (Beispiel 52 und Kommentar dazu).

Das Oktoton as bis a, dem die Töne $c$, $f$ fehlen (T. 30-45), wird in der Coda vervollständigt. Das fis des Heptatons a bis dis plus c (T. 57; Beispiel 67, vierter Akkord) bleibt in Takt 69 und 71 auffällig allein liegen. Darauf folgt die plagale Schlusswendung $f_{-C}($ T. 78/79), die das Oktoton ergänzt. Vgl. dazu die Vorandeutung $f$-c auf der Wende Takt 49/50 und Simons Beispiel 55 NB mit Kommentar.

Die beiden Tonfelder des Ganzen können also folgendermaßen dargestellt werden:

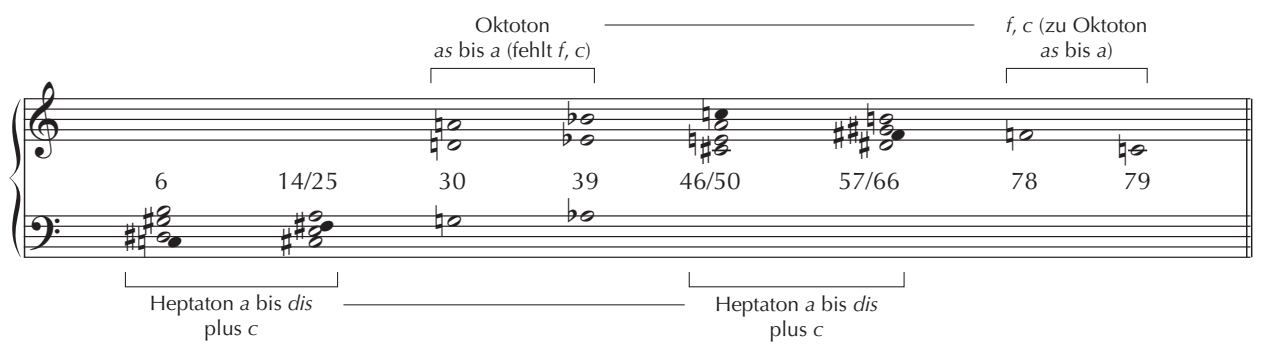

Beispiel 74

Der zweite Klang von Beispiel 74 kommt als Teil eines Quintenfeldes zustande (dazu vgl. Kommentar zu Beispiel 67), er kann aber auch als Schnittmenge des Pentatons a bis cis mit der Tonika aufgefasst werden. Daher wird im Detail tatsächlich die Tonika vollständig ausgeführt (d. h. es werden die Töne $c$, ais, g, dis hinzugefügt, vgl. Beispiel 40 und - analog - Beispiel 58, in dem die Töne $d, c$, a, f zum Klang gis/h/dis/fis hinzugefügt 
werden. Es handelt sich jeweils um eine effizierte vollständige Funktion: Tonika bzw. Subdominante). Simons Beispiel 47 beschreibt etwas wie eine Modulation vom letzten Klang von Beispiel 70 (T. 35) zum vierten Klang von Beispiel 74 (T. 39).

Mehr als in vieler älterer Musik scheint in diesen beiden Kompositionen Bartóks zumindest das erste Tonfeld des Ganzen mehr eine Vororganisation der Töne insgesamt zu sein als aus bestimmten lokalisierbaren Tönen zu bestehen. So liefern in Staccato die vier Gestalten des unvariierten Grundmotivs (Grundgestalt und Umkehrung T. 3/4, 11/12, 12, 17) die beiden Pentatone (aus denen maßgeblich das erste Tonfeld besteht), aber andere Tonfolgen (z.B. g-a-h T. 9, der Quartraum a- $d^{1}$ der Oberstimme T. 4-7) entspringen ebenso diesen Pentatonen. In Geteilte Arpeggien hätte es wenig Sinn, z. B. das cis des Heptatons lokalisieren zu wollen: Takt 10, 14-26, alle sind es gleichermaßen. Das jeweils zweite Tonfeld des Ganzen ist in beiden Kompositionen dagegen genau lokalisiert. Es wird lohnen, den Bezug zu untersuchen von Tonfeldern, die das Ganze definieren, zu detaillierten Tonfeldern und schließlich zu Tonfeldern, die zwischen diesen Ebenen stehen und sie verbinden.

\section{Literatur}

Bartók, Béla (1976), »Harvard lectures«, in: Béla Bartók, Essays, hg. von Benjamin Suchoff, London: Faber \& Faber, 354-392.

Gárdonyi, Zsolt und Hubert Nordhoff (2002), Harmonik, 2. Aufl. Wolfenbüttel: Möseler.

Gollin, Edward (2007), »Multi-Aggregate Cycles and Multi-Aggregate Serial Techniques in the Music of Béla Bartók«, Music Theory Spectrum 29, 143-176.

Haas, Bernhard (2004), Die neue Tonalität von Schubert bis Webern. Hören und Analysieren nach Albert Simon, Wilhelmshaven: Noetzel.

Lendvai, Ernö (1995), Symmetrien in der Musik. Einführung in die musikalische Semantik, übers. von Siglind Bruhn, Kecskemét und Wien: Kodály Institut und Universal Edition. 